\title{
The effective Standard Model after LHC Run I
}

\author{
John Ellis, ${ }^{a, b}$ Verónica Sanz ${ }^{c}$ and Tevong You ${ }^{a}$ \\ ${ }^{a}$ Theoretical Particle Physics and Cosmology Group, \\ Physics Department, King's College London, \\ London WC2R 2LS, U.K. \\ ${ }^{b}$ TH Division, Physics Department, CERN, \\ CH-1211 Geneva 23, Switzerland \\ ${ }^{c}$ Department of Physics and Astronomy, University of Sussex, \\ Brighton BN1 9QH, U.K. \\ E-mail: john.ellis@cern.ch, v.sanz@sussex.ac.uk, tevong.you@kcl.ac.uk
}

\begin{abstract}
We treat the Standard Model as the low-energy limit of an effective field theory that incorporates higher-dimensional operators to capture the effects of decoupled new physics. We consider the constraints imposed on the coefficients of dimension-6 operators by electroweak precision tests (EWPTs), applying a framework for the effects of dimension6 operators on electroweak precision tests that is more general than the standard $S, T$ formalism, and use measurements of Higgs couplings and the kinematics of associated Higgs production at the Tevatron and LHC, as well as triple-gauge couplings at the LHC. We highlight the complementarity between EWPTs, Tevatron and LHC measurements in obtaining model-independent limits on the effective Standard Model after LHC Run 1. We illustrate the combined constraints with the example of the two-Higgs doublet model.
\end{abstract}

Keywords: Higgs Physics, Beyond Standard Model, Effective field theories

ARXIV EPRINT: 1410.7703 


\section{Contents}

1 Introduction 1

2 Electroweak Precision Tests at LEP 2

2.1 The expansion formalism 3

2.2 Dimension-6 operators in EWPTs 5

3 Triple-gauge and Higgs couplings at the LHC 9

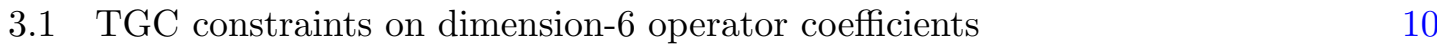

3.2 Inclusion of Higgs associated production constraints 13

4 Application to the Two-Higgs Doublet Model 14

$\begin{array}{llr}5 & \text { Conclusions } & 17\end{array}$

$\begin{array}{ll}\text { A Kinematics and the validity of the effective field theory } & 18\end{array}$

\section{Introduction}

Run 1 of the LHC has taken probes of the Standard Model to a new level, not only by the discovery of the Higgs boson $H(125)[1,2]$ and the absence of other new particles, but also via the new constraints imposed on the couplings of vector bosons and the top quark [3-41]. Now is an appropriate time to assess the global constraints placed on possible new physics by LHC Run 1 in conjunction with the Tevatron, LEP and other experiments. In view of the kinematic reach of the LHC, it is natural to suppose that the threshold for any new physics may lie substantially above the masses of the Standard Model particles. In this case, the new physics may be analyzed in the decoupling limit [42], and its effects may be parameterized in terms of higher-dimensional operators composed of Standard Model fields [43]. Using the equations of motions reduces the number of independent operators [44-49], with a complete non-redundant set first categorised in [50].

This is the effective Standard Model approach adopted in a large number of recent papers $^{1}$ [53-83], and there have been many analyses of the constraints imposed on new physics via upper limits on the coefficients of a complete dimension- 6 operator basis [8489], in particular. Several different classes of measurements make important contributions to these constraints. LEP and other experiments contribute via electroweak precision tests (EWPTs) [90], which are often presented as constraints on the $S$ and $T$ parameters that are defined in terms of oblique radiative corrections due to vacuum polarization diagrams, and

\footnotetext{
${ }^{1}$ For earlier studies of dimension- 6 operators in triple-gauge couplings and Higgs physics see for example $[51,52]$.
} 
via measurements of triple-gauge couplings (TGCs). The Tevatron experiments contribute via measurements of (constraints on) production of the Higgs boson $H$ in association with massive gauge bosons $V=W^{ \pm}, Z^{0}[91]$. Finally, the LHC experiments contribute via many Higgs measurements including signal strengths [92, 93], branching ratios and kinematic distributions [94], and also via TGC measurements [95-97].

We demonstrated in previous work [89] the power of the constraints provided by measurements of kinematic distributions in $V+H$ production at the Tevatron and the LHC, showing that measurements of the $V+H$ invariant mass $M_{V H}$ at the Tevatron and the transverse momentum $p_{T}^{V}$ at the LHC could close off a 'blind' direction in the parameter space of dimension- 6 operator coefficients that had been allowed by previous analyses of LEP and LHC data [98]. ${ }^{2}$ Subsequently, new data on TGCs from LHC running at $8 \mathrm{TeV}$ have been published [95-97]. In this paper we make the first complete analysis of the data from LHC Run 1 and the Tevatron, in combination with the EWPT constraints, considering only $\mathrm{CP}$-even operators and assuming minimal flavour violation. We consider a complete set of operators in a non-redundant basis, and the 95\% CL ranges that we find for their coefficients are listed in tables 1 and 2 .

We confirm previous findings that the EWPTs place very strong constraints on certain (combinations of) operator coefficients. On the other hand, we also find that the Higgs observables (signal strengths and associated production kinematics) and the TGC measurements at the LHC also have complementary rôles to play. Some operator coefficients are better constrained by the TGC data, and some by the Higgs data. One coefficient in particular only affects TGCs and nothing else. Only their combination provides a complete picture of the constraints on the dimension-6 operator coefficients after LHC Run 1.

The outline of this paper is as follows. In section 2 we discuss the EWPTs, first reviewing a general expansion formalism for EWPTs, and then demonstrating that it reproduces the constraints on the vacuum polarization parameters $S$ and $T$ found in other analyses before illustrating its use in capturing the effects of a complete basis of dimension-6 operators. In section 3 we discuss the constraints imposed by measurements of Higgs couplings, associated Higgs production kinematics and TGCs at the LHC, demonstrating their complementarity. Section 4 illustrates the application of these combined constraints on the coefficients of dimension- 6 operators to the two-Higgs-doublet model (2HDM). Section 5 summarizes our conclusions and assesses some future prospects, and an appendix discusses aspects of kinematics and the applicability of effective field theory in our analysis.

\section{Electroweak Precision Tests at LEP}

Electroweak precision tests (EWPTs), particularly those provided by LEP, are amongst the most sensitive observables for constraining new physics beyond the Standard Model. EWPTs are typically summarized via constraints on the $S$ and $T$ parameters $[99,100]$ and their generalization to include the $W$ and $Y$ parameters $[101,102]$ that are relevant for custodially-symmetric and weak isospin-preserving new physics, which characterize the

\footnotetext{
${ }^{2}$ Contribution to G. Brooijmans et al., Les Houches 2013: Physics at TeV Colliders: New Physics Working Group Report, arXiv:1405.1617.
} 
Standard Model vector boson self-energy corrections. ${ }^{3}$ If new physics affects only the Standard Model gauge sector and does not couple directly to Standard Model fermions, this approach may be sufficient for placing bounds on such 'universal' models, but the effective Standard Model also includes fermionic operators that affect electroweak precision tests. Thus a more general framework is required to capture all the possible effects of decoupled new physics in a model-independent way.

There have been many studies considering individual or subsets of bounds for all dimension-6 operators entering in EWPTs, for example [104, 105], and full analyses including simultaneously a complete basis of dimension-6 operators affecting these EWPTs have been performed in [86-88], but a full calculation of the effects of propagation of corrections to input observables and self-energies as well as direct contributions to observables was needed in each different basis. Here we employ instead the recent expansion formalism of [106], which separates the calculation of the corrections' effects on the EWPT observables and the calculations of the contributions to the corrections from new physics. This framework facilitates any $\chi^{2}$ analysis that seeks to go beyond the $S, T$ parametrization and renders more transparent the origin of the effects from each operator.

\subsection{The expansion formalism}

For convenience, we briefly summarize here the analysis of [106]. The principle is that, given the Standard Model with Lagrangian parameters $p_{\mathrm{SM}} \equiv\left\{g, g^{\prime}, g_{s}, y_{t}, v, \lambda\right\}$, one may calculate theoretical values $\hat{\mathcal{O}}_{i}^{\text {th }}\left(p_{\mathrm{SM}}\right)$ for the observables

$$
\hat{\mathcal{O}}_{i} \equiv\left\{m_{Z}, G_{F}, \alpha\left(m_{Z}\right), m_{t}, \alpha_{s}, m_{H}, m_{W}, \Gamma_{l}, \Gamma_{q}, \sigma_{\text {had }}, R_{l}, \sin ^{2} \theta_{\text {eff }}, A_{f}, A_{F B}^{f}, \ldots\right\}
$$

that are measured by experiments with errors $\Delta \hat{\mathcal{O}}_{i}^{\text {exp }}$. To compare the theoretical predictions $\hat{\mathcal{O}}_{i}^{\text {th }}\left(p_{\mathrm{SM}}\right)$ with the experimental measurements, $\hat{\mathcal{O}}_{i}^{\exp }$, we must first choose 6 of these observables as 'input' observables $\hat{\mathcal{O}}_{i^{\prime}}$, typically the most precisely measured ones, ${ }^{4}$ such as

$$
\hat{\mathcal{O}}_{i^{\prime}} \equiv\left\{m_{Z}, G_{F}, \alpha\left(m_{Z}\right), m_{t}, \alpha_{s}, m_{H}\right\}
$$

These assign values $p_{\mathrm{SM}}^{\mathrm{ref}}$ to the Lagrangian parameters such that the $\hat{\mathcal{O}}_{i^{\prime}}^{\text {th }}\left(p_{\mathrm{SM}}^{\mathrm{ref}}\right)$ agree well with measurements, and numerical values for the other 'output' observables can then be obtained in terms of $p_{\mathrm{SM}}^{\mathrm{ref}}$.

In the presence of new physics characterized by parameters $p_{\alpha}$, the theoretical expressions for the observables are modified by a correction $\delta^{\mathrm{NP}} \hat{\mathcal{O}}_{i}\left(p_{\mathrm{SM}}, p_{\alpha}\right)$ :

$$
\hat{\mathcal{O}}_{i}^{\text {th }}\left(p_{\mathrm{SM}}, p_{\alpha}\right)=\hat{\mathcal{O}}_{i}^{\mathrm{SM}}\left(p_{\mathrm{SM}}\right)+\delta^{\mathrm{NP}} \hat{\mathcal{O}}_{i}\left(p_{\mathrm{SM}}, p_{\alpha}\right)
$$

Since the relations between input observables and Lagrangian parameters are modified in general, a different $p_{\mathrm{SM}}^{\mathrm{ref}}$ value would normally be preferred to compensate for non-zero

\footnotetext{
${ }^{3}$ See also [103] for another parametrisation of EWPT fits that includes vertex corrections in a set of $\epsilon$ parameters.

${ }^{4}$ Another convenient choice of input observables is to use $m_{W}$ instead of $G_{F}$ [107].
} 
values of $p_{\alpha}$ so as to remain in agreement with experiment. This may be quantified by a $\chi^{2}$ analysis that varies the parameters $\left(p_{\mathrm{SM}}, p_{\alpha}\right)$ so as to minimize the function

$$
\chi^{2}\left(p_{\mathrm{SM}}, p_{\alpha}\right)=\sum_{i, j}\left(\hat{\mathcal{O}}_{i}^{\mathrm{th}}-\hat{\mathcal{O}}_{i}^{\exp }\right)\left(\sigma^{2}\right)_{i j}^{-1}\left(\hat{\mathcal{O}}_{j}^{\mathrm{th}}-\hat{\mathcal{O}}_{j}^{\exp }\right), \quad\left(\sigma^{2}\right)_{i j}=\Delta \hat{\mathcal{O}}_{i}^{\exp } \rho_{i j} \Delta \hat{\mathcal{O}}_{j}^{\exp }
$$

where $\rho_{i j}$ is the correlation matrix.

To avoid recomputing the full expression $\hat{\mathcal{O}}_{i}^{\text {th }}\left(p_{\mathrm{SM}}, p_{\alpha}\right)$ for each value of $p_{\mathrm{SM}}$ and $p_{\alpha}$, the expansion formalism involves expanding about the Standard Model reference values for the Lagrangian parameters:

$$
\begin{aligned}
\hat{\mathcal{O}}_{i}^{\mathrm{SM}}\left(p_{\mathrm{SM}}\right) & =\hat{\mathcal{O}}_{i}^{\mathrm{SM}}\left(p_{\mathrm{SM}}^{\mathrm{ref}}\right)+\sum_{p_{\mathrm{SM}}} \frac{\partial \hat{\mathcal{O}}_{i}^{\mathrm{SM}}}{\partial p_{\mathrm{SM}}}\left(p_{\mathrm{SM}}-p_{\mathrm{SM}}^{\mathrm{ref}}\right)+\ldots \\
& \simeq \hat{\mathcal{O}}_{i}^{\mathrm{ref}}\left[1+\bar{\delta}^{\mathrm{SM}} \hat{\mathcal{O}}_{i}\left(p_{\mathrm{SM}}\right)\right]
\end{aligned}
$$

where $\hat{\mathcal{O}}_{i}^{\text {ref }} \equiv \hat{\mathcal{O}}_{i}^{\mathrm{SM}}\left(p_{\mathrm{SM}}^{\mathrm{ref}}\right), \bar{\delta}^{\mathrm{SM}} \hat{\mathcal{O}}_{i}\left(p_{\mathrm{SM}}\right)=\sum_{p_{\mathrm{SM}}} G_{i p_{\mathrm{SM}}} \bar{\delta} p_{\mathrm{SM}}$, and the quantities $G_{i k^{\prime}} \equiv$ $\frac{p_{\mathrm{SM}}^{\text {ref }}}{\overline{\mathcal{O}}_{i}^{\text {ref }}} \frac{\partial \hat{\mathcal{O}}_{i}^{\mathrm{SM}}}{\partial p_{\mathrm{SM}}}$ are expansion coefficients that need only to be calculated once. Here $\bar{\delta} p_{\mathrm{SM}} \equiv$ $\left(p_{\mathrm{SM}}-p_{\mathrm{SM}}^{\mathrm{ref}}\right) / p_{\mathrm{SM}}^{\mathrm{ref}}$, and the fractional shift $\bar{\delta}$ is defined in general as $\bar{\delta} \hat{\mathcal{O}}_{i} \equiv\left(\hat{\mathcal{O}}_{i}-\hat{\mathcal{O}}_{i}^{\text {ref }}\right) / \hat{\mathcal{O}}_{i}^{\text {ref }}$. The reference values for the SM observables are taken from table 1 of [106], to which we refer the reader for more details on the numerical calculation including the higher-order loop corrections, which were obtained using ZFITTER [108]. This is also used for the numerical differentiation involved in evaluating the expansion coefficients, which assumes that the new physics contribution factorizes out of the SM loop expansion.

Furthermore, to emphasize that the $p_{\mathrm{SM}}$ are not directly measurable, but are determined from the input observables $\hat{\mathcal{O}}_{i^{\prime}}$, we note that the Lagrangian parameters can be eliminated in favour of the input observables by inverting the relation $\bar{\delta}^{\mathrm{SM}} \hat{\mathcal{O}}_{i^{\prime}}=$ $\sum_{p_{\mathrm{SM}}} G_{i^{\prime} p_{\mathrm{SM}}} \bar{\delta} p_{\mathrm{SM}}$, so that

$$
\bar{\delta}^{\mathrm{SM}} \hat{\mathcal{O}}_{i}=\sum_{i^{\prime}} G_{i p_{\mathrm{SM}}}\left(\sum_{p_{\mathrm{SM}}}\left(G^{-1}\right)_{p_{\mathrm{SM}} i^{\prime}} \bar{\delta}^{\mathrm{SM}} \hat{\mathcal{O}}_{i^{\prime}}\right)=\sum_{i^{\prime}} d_{i i^{\prime}} \bar{\delta}^{\mathrm{SM}} \hat{\mathcal{O}}_{i^{\prime}}
$$

The expansion coefficients for the output observables in terms of input observables are then given by the matrix $d_{i i^{\prime}} \equiv \sum_{p_{\mathrm{SM}}} G_{i p_{\mathrm{SM}}}\left(G^{-1}\right)_{p_{\mathrm{SM}} i^{\prime}}$.

The theoretical predictions for the output observables can now be written as $\hat{\mathcal{O}}_{i}^{\text {th }}=$ $\hat{\mathcal{O}}_{i}^{\text {ref }}\left(1+\bar{\delta} \hat{\mathcal{O}}_{i}^{\text {th }}\right)$, with

$$
\bar{\delta} \hat{\mathcal{O}}_{i}^{\text {th }}=\sum_{i^{\prime}} d_{i i^{\prime}} \bar{\delta}^{\mathrm{SM}} \hat{\mathcal{O}}_{i^{\prime}}+\xi_{i}=\sum_{i^{\prime}} d_{i i^{\prime}}\left(\bar{\delta} \hat{\mathcal{O}}_{i^{\prime}}^{\text {th }}-\xi_{i^{\prime}}\right)+\xi_{i},
$$

where we used $\bar{\delta} \hat{\mathcal{O}}_{i^{\prime}}^{\mathrm{SM}}=\bar{\delta} \hat{\mathcal{O}}_{i^{\prime}}^{\text {th }}-\xi_{i^{\prime}}$ and defined $\xi_{i} \equiv \delta^{\mathrm{NP}} \hat{\mathcal{O}}_{i} / \hat{\mathcal{O}}_{i}^{\text {ref }}$. The $d_{i i^{\prime}}$ matrix is pre-calculated and encapsulates the dependence of each output observable on each input observable, so that one needs only to plug in the contribution due to new physics that affect the input observables, $\xi_{i^{\prime}}$, and those that directly affect the output observables, $\xi_{i}$. We note that, for the case of vector boson self-energy corrections, the $\pi_{V V} \equiv$ 


\begin{tabular}{|c|c|c|c|}
\hline Operator & Coefficient & \multicolumn{2}{|c|}{ LEP Constraints } \\
\hline $\mathcal{O}_{W}=\frac{i g}{2}\left(H^{\dagger} \sigma^{a} \stackrel{\leftrightarrow}{D^{\mu}} H\right) D^{\nu} W_{\mu \nu}^{a}$ & $m_{W}^{2}\left(c_{W}+c_{B}\right)$ & $(-0.00055,0.0005)$ & $(-0.0033,0.0018)$ \\
$\mathcal{O}_{B}=\frac{i g^{\prime}}{2}\left(H^{\dagger} \stackrel{\leftrightarrow}{D^{\mu}} H\right) \partial^{\nu} B_{\mu \nu}$ & & & \\
\hline $\mathcal{O}_{T}=\frac{1}{2}\left(H^{\dagger} \stackrel{\leftrightarrow}{D_{\mu}} H\right)^{2}$ & $\frac{v^{2}}{\Lambda^{2}} c_{T}$ & $(0,0.001)$ & $(-0.0043,0.0033)$ \\
\hline $\mathcal{O}_{L L}^{(3) l}=\left(\bar{L}_{L} \sigma^{a} \gamma^{\mu} L_{L}\right)\left(\bar{L}_{L} \sigma^{a} \gamma_{\mu} L_{L}\right)$ & $\frac{v^{2}}{\Lambda^{2}} c_{L L}^{(3) l}$ & $(0,0.001)$ & $(-0.0013,0.00075)$ \\
\hline $\mathcal{O}_{R}^{e}=\left(i H^{\dagger} \stackrel{\leftrightarrow}{D_{\mu}} H\right)\left(\bar{e}_{R} \gamma^{\mu} e_{R}\right)$ & $\frac{v^{2}}{\Lambda^{2}} c_{R}^{e}$ & $(-0.0015,0.0005)$ & $(-0.0018,0.00025)$ \\
\hline $\mathcal{O}_{R}^{u}=\left(i H^{\dagger} \stackrel{\leftrightarrow}{D_{\mu}} H\right)\left(\bar{u}_{R} \gamma^{\mu} u_{R}\right)$ & $\frac{v^{2}}{\Lambda^{2}} c_{R}^{u}$ & $(-0.0035,0.005)$ & $(-0.011,0.011)$ \\
\hline $\mathcal{O}_{R}^{d}=\left(i H^{\dagger} \stackrel{\leftrightarrow}{D_{\mu}} H\right)\left(\bar{d}_{R} \gamma^{\mu} d_{R}\right)$ & $\frac{v^{2}}{\Lambda^{2}} c_{R}^{d}$ & $(-0.0075,0.0035)$ & $(-0.042,0.0044)$ \\
\hline $\mathcal{O}_{L}^{(3) q}=\left(i H^{\dagger} \sigma^{a} \stackrel{\leftrightarrow}{D_{\mu}} H\right)\left(\bar{Q}_{L} \sigma^{a} \gamma^{\mu} Q_{L}\right)$ & $\frac{v^{2}}{\Lambda^{2}} c_{L}^{(3) q}$ & $(-0.0005,0.001)$ & $(-0.0044,0.0044)$ \\
\hline $\mathcal{O}_{L}^{q}=\left(i H^{\dagger} \stackrel{\leftrightarrow}{D_{\mu}} H\right)\left(\bar{Q}_{L} \gamma^{\mu} Q_{L}\right)$ & $\frac{v^{2}}{\Lambda^{2}} c_{L}^{q}$ & $(-0.0015,0.003)$ & $(-0.0019,0.0069)$ \\
\hline
\end{tabular}

Table 1. List of operators and coefficients in our basis entering in EWPTs at LEP, together with $95 \%$ CL bounds when individual coefficients are switched on one at a time, and marginalized in a simultaneous fit. For the first four coefficients we report the constraints from the leptonic observables, while the remaining coefficients also include the hadronic observables.

$\left\{\pi_{Z Z}, \pi_{Z Z}^{\prime}, \pi_{\gamma Z}, \pi_{\gamma \gamma}^{\prime}, \pi_{+-}, \pi_{W W}^{0}\right\}$ are defined as in [106], and the contributions to output observables through $\xi_{i^{\prime}}$ and $\xi_{i}$ are summarized by the given $b_{i, V V}$ coefficients. We then have

$$
\bar{\delta} \hat{\mathcal{O}}_{i}^{\text {th }}=\sum_{i^{\prime}} d_{i i^{\prime}} \bar{\delta} \hat{\mathcal{O}}_{i^{\prime}}^{\text {th }}+\bar{\delta}^{\mathrm{NP}} \hat{\mathcal{O}}_{i}
$$

where

$$
\bar{\delta}^{\mathrm{NP}} \hat{\mathcal{O}}_{i} \equiv \xi_{i}-\sum_{i^{\prime}} d_{i i^{\prime}} \xi_{i^{\prime}}+\sum_{V V} b_{i, V V} \delta^{\mathrm{NP}} \pi_{V V},
$$

and it remains only to determine the $\xi_{i^{\prime}}, \xi_{i}$ and $\delta^{\mathrm{NP}} \pi_{V V}$ from the dimension- 6 operators in the effective Standard Model.

\subsection{Dimension-6 operators in EWPTs}

We begin with the familiar $S, T$ parameters before generalizing to a complete dimension- 6 operator basis. The universal parts of new physics contributions are often parametrized as oblique corrections to vector boson self-energies, which can be written in terms of gauge eigenstates as

$$
\mathcal{L}_{\mathrm{VV}}=-W^{+\mu} \pi_{+-}\left(p^{2}\right) W_{\mu}^{-}-\frac{1}{2} W^{3^{\mu}} \pi_{33}\left(p^{2}\right) W_{\mu}^{3}-W^{3^{\mu}} \pi_{3 B}\left(p^{2}\right) B_{\mu}-\frac{1}{2} B^{\mu} \pi_{B B}\left(p^{2}\right) B_{\mu},
$$


where $\pi_{V V}\left(p^{2}\right)=\pi_{V V}^{\mathrm{SM}}\left(p^{2}\right)+\delta \pi_{V V}\left(p^{2}\right)$. Making a Taylor expansion at the quadratic order to which dimension- 6 operators can contribute:

$$
\pi_{V V}\left(p^{2}\right)=\pi_{V V}(0)+p^{2} \pi_{V V}^{\prime}(0)+\frac{1}{2}\left(p^{2}\right)^{2} \pi_{V V}^{\prime \prime}(0)+\ldots
$$

the usual $\hat{S}$ and $\hat{T}$ parameters ${ }^{5}$ can be defined as

$$
\hat{S} \equiv \frac{g}{g^{\prime}} \frac{\pi_{3 B}^{\prime}(0)}{\pi_{+-}^{\prime}(0)}, \quad \hat{T} \equiv \frac{\pi_{+-}(0)-\pi_{33}(0)}{\pi_{+-}(0)}
$$

Since $\mathrm{U}(1)_{Q}$ symmetry is conserved, which requires $\pi_{\gamma \gamma}(0)$ and $\pi_{\gamma Z}(0)$ to vanish by gauge invariance, the following relations must hold:

$$
\begin{aligned}
g^{\prime 2} \pi_{33}(0)+g^{2} \pi_{B B}(0)+2 g g^{\prime} \pi_{3 B}(0) & =0 \\
g \pi_{B B}(0)+g^{\prime} \pi_{3 B}(0) & =0 .
\end{aligned}
$$

After normalizing the $W^{ \pm}$and $B$ fields so that the kinetic terms are canonical and $\pi_{+-}(0)=$ $-m_{W}^{2}$, we obtain the following $\hat{S}$ and $\hat{T}$ corrections in the gauge mass eigenstates for the quantities $\delta^{\mathrm{NP}} \pi_{V V}$ defined in [106]:

$$
\begin{aligned}
& \delta^{\mathrm{NP}} \pi_{Z Z}=-\hat{T}+2 \hat{S} \sin ^{2} \theta_{W} \\
& \delta^{\mathrm{NP}} \pi_{Z Z}^{\prime}=2 \hat{S} \sin ^{2} \theta_{W} \\
& \delta^{\mathrm{NP}} \pi_{\gamma Z}=-\hat{S} \cos 2 \theta_{W} \tan \theta_{W} \\
& \delta^{\mathrm{NP}} \pi_{\gamma \gamma}^{\prime}=-2 \hat{S} \sin ^{2} \theta_{W} .
\end{aligned}
$$

Inserting these expressions into (2.1) and performing a $\chi^{2}$ analysis in the expansion formalism, using as output observables the EWPTs at the $Z$ peak and the $W$ mass:

$\hat{\mathcal{O}}_{i}=\left\{\Gamma_{Z}, \sigma_{\mathrm{had}}^{0}, R_{e}^{0}, R_{\mu}^{0}, R_{\tau}^{0}, A_{\mathrm{FB}}^{0, e}, \sin ^{2} \theta_{\mathrm{eff}}^{e}, R_{b}^{0}, R_{c}^{0}, A_{\mathrm{FB}}^{0, b}, A_{\mathrm{FB}}^{0, c}, A_{b}, A_{c}, \sin ^{2} \theta_{\mathrm{eff}}^{b}, \sin ^{2} \theta_{\mathrm{eff}}^{c}, m_{W}\right\}$,

we obtain the $68 \%, 95 \%$, and $99 \% \mathrm{CL}$ allowed regions for $S$ vs $T$ shown in figure 1, denoted by dotted, dashed and solid contours respectively. We treat the observables as uncorrelated but have checked that including the correlation matrix, for example in the leptonic subset as given in [90], does not affect substantially our results, which agree reasonably closely with those of [109].

The $\hat{S}$ and $\hat{T}$ parameters are equivalent to a subset of the full set of dimension- 6 operators that can affect the EWPTs. In a redundant basis those entering in oblique corrections to vector boson self-energies are

$$
\mathcal{L}_{\text {dim- } 6} \supset \frac{\bar{c}_{W B}}{m_{W}^{2}} \mathcal{O}_{W B}+\frac{\bar{c}_{W}}{m_{W}^{2}} \mathcal{O}_{W}+\frac{\bar{c}_{B}}{m_{W}^{2}} \mathcal{O}_{B}+\frac{\bar{c}_{T}}{v^{2}} \mathcal{O}_{T}+\frac{\bar{c}_{2 W}}{m_{W}^{2}} \mathcal{O}_{2 W}+\frac{\bar{c}_{2 B}}{m_{W}^{2}} \mathcal{O}_{2 B},
$$

\footnotetext{
${ }^{5}$ These are related to the $S$ and $T$ parameters defined in $[99,100]$ via $S=\frac{4 \sin ^{2} \theta_{W}}{\alpha\left(m_{Z}\right)} \hat{S} \approx 119 \hat{S}$ and $T=\frac{1}{\alpha\left(m_{Z}\right)} \hat{T} \approx 129 \hat{T}$.
} 


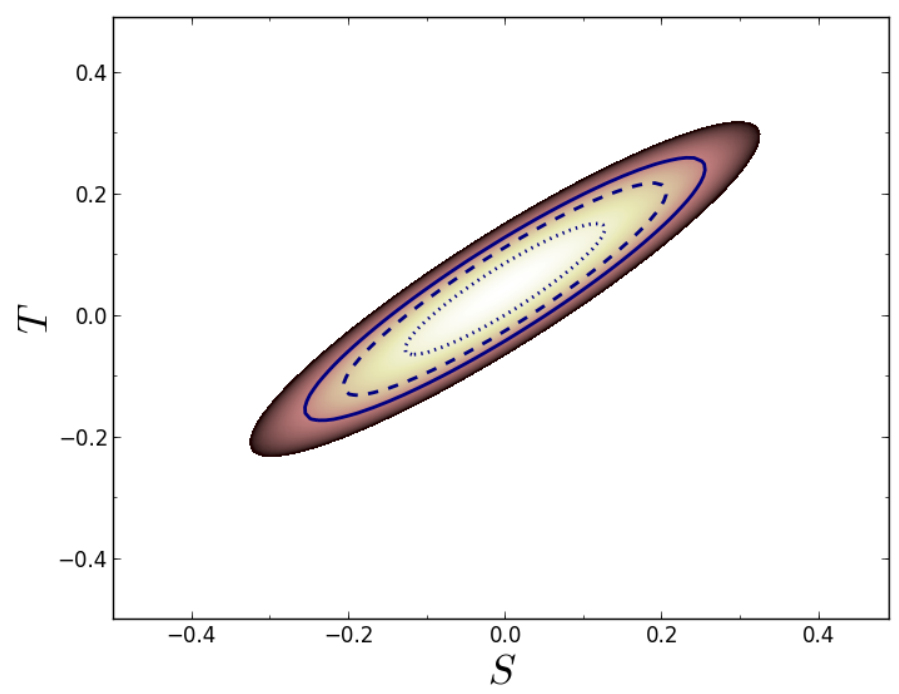

Figure 1. Results of a $\chi^{2}$ analysis of $S T$ parameters in EWPTs using the expansion formalism of [106]. The dotted, dashed and solid contours denote the regions allowed at the $68 \%, 95 \%$, and $99 \%$ CL, respectively, which may be compared with those of [109].

while those that affect the leptonic and hadronic $Z$-pole measurements directly through modifications to the gauge boson-fermion couplings are

$$
\mathcal{L}_{\operatorname{dim}-6} \supset \sum_{f_{L}}\left(\frac{\bar{c}_{f_{L}}}{v^{2}} \mathcal{O}_{f_{L}}+\frac{\bar{c}_{f_{L}}^{(3)}}{v^{2}} \mathcal{O}_{f_{L}}^{(3)}\right)+\sum_{f_{R}} \frac{\bar{c}_{f_{R}}}{v^{2}} \mathcal{O}_{f_{R}} .
$$

The sum is over the left-handed lepton and quark doublets, $f_{L} \equiv L_{L}, Q_{L}$, and right-handed lepton and quark singlets, $f_{R} \equiv e_{R}, u_{R}, d_{R}$, and we assume minimal flavour violation. The Fermi constant $G_{F}$ defined by the muon lifetime, which we take as an input observable, is modified by $\bar{c}_{f_{L}}^{(3)}$ as well as the four-fermion operator $\mathcal{O}_{L L}^{(3) l}$ :

$$
\mathcal{L}_{\text {dim- }-6} \supset \frac{\bar{c}_{L L}^{(3) l}}{v^{2}} \mathcal{O}_{L L}^{(3) l} .
$$

We note that the coefficients are defined such that

$$
\bar{c} \equiv c \frac{M^{2}}{\Lambda^{2}},
$$

where $M \equiv v, m_{W}$ depending on the operator normalization, and $c \sim g_{\mathrm{NP}}^{2}$ is a coefficient proportional to a new physics coupling $g_{\mathrm{NP}}$ defined at the scale $M$. These are related to the coefficients at the new physics scale through RGE equations [110-117].

These operators form a redundant basis that is reducible through field redefinitions, or equivalently the equations of motion, that have no effect on the S-matrix [44-49]. Following [88], we may eliminate the operators $\mathcal{O}_{L_{L}}, \mathcal{O}_{L_{L}}^{(3)}$ that affect the left-handed leptonic $Z$ couplings, and the operators $\mathcal{O}_{2 W}, \mathcal{O}_{2 B}, \mathcal{O}_{2 G}$ corresponding to the $Y, W$ and $Z$ parameters $[101,102]$ in the generalization of the universal oblique parameters. ${ }^{6}$ The coefficients

\footnotetext{
${ }^{6}$ The $U, V$ and $X$ parameters correspond to higher-dimensional operators.
} 
$\bar{c}_{W B}$ and the combination $\bar{c}_{W}+\bar{c}_{B}$ are related to the $\hat{S}$ parameter, and we eliminate the former using the identity

$$
\mathcal{O}_{B}=\mathcal{O}_{H B}+\frac{1}{4} \mathcal{O}_{B B}+\frac{1}{4} \mathcal{O}_{W B}
$$

The operators $\mathcal{O}_{H B}, \mathcal{O}_{B B}$ affect Higgs physics and triple-gauge couplings, as we shall see in the next section. Finally, the $\hat{T}$ parameter is equivalent to the $\bar{c}_{T}$ coefficient. This choice of basis minimises the correlation of operator combinations among EWPT and LHC measurements. These operators are listed in table 1 , and the remaining operators eliminated from our basis are defined in [118].

The corrections to the self-energies are then as in (2.5), with $\hat{S}=\bar{c}_{W}+\bar{c}_{B}$ and $\hat{T}=\bar{c}_{T}$. We also have the input observable correction

$$
\xi_{G_{F}}=-2 \bar{c}_{L L}^{(3) l}
$$

and direct contributions to the output observables,

$$
\begin{aligned}
\xi_{\Gamma_{Z}} & =\frac{\Gamma_{Z}^{l}}{\Gamma_{Z}} \xi_{\Gamma_{Z}^{l}}+\frac{\Gamma_{Z}^{\mathrm{had}}}{\Gamma_{Z}} \xi_{\Gamma_{Z}^{\mathrm{had}}}, \\
\xi_{\sigma_{\mathrm{had}}^{0}} & =\xi_{\Gamma_{Z}^{e}}+\xi_{\Gamma_{Z}^{\mathrm{had}}}-2 \xi_{\Gamma_{Z}} \\
\xi_{R_{l}} & =\xi_{\Gamma_{Z}^{\mathrm{had}}}-\xi_{\Gamma_{Z}^{l}} \\
\xi_{R_{q}} & =\xi_{\Gamma_{Z}^{q}}-\xi_{\Gamma_{Z}^{\mathrm{had}}} \\
\xi_{A_{\mathrm{FB}}^{0, f}} & =\xi_{A_{e}}+\xi_{A_{f}},
\end{aligned}
$$

which can be written in terms of shifts to the $Z$-fermion couplings,

$$
\begin{aligned}
\xi_{A_{f}} & =\frac{4\left(g_{Z}^{f_{L}}\right)^{2}\left(g_{Z}^{f_{R}}\right)^{2}}{\left(g_{Z}^{f_{L}}\right)^{4}-\left(g_{Z}^{f_{R}}\right)^{4}}\left(\xi_{g_{Z}^{f_{L}}}-\xi_{g_{Z}^{f_{R}}}\right), \\
\xi_{\Gamma_{Z}^{f}} & =\frac{2\left(g_{Z}^{f_{L}}\right)^{2}}{\left(g_{Z}^{f_{L}}\right)^{2}+\left(g_{Z}^{f_{R}}\right)^{2}} \xi_{g_{Z}^{f_{L}}}+\frac{2\left(g_{Z}^{f_{R}}\right)^{2}}{\left(g_{Z}^{f_{L}}\right)^{2}+\left(g_{Z}^{f_{R}}\right)^{2}} \xi_{g_{Z}^{f_{R}}},
\end{aligned}
$$

where

$$
\xi_{g_{Z}^{f_{L}}}=\frac{1}{g_{Z}^{f_{L}}}\left(T_{f}^{3} \bar{c}_{f_{L}}^{(3)}-\frac{\bar{c}_{f_{L}}}{2}\right), \quad \xi_{g_{Z}^{f_{R}}}=-\frac{\bar{c}_{f_{R}}}{2 g_{Z}^{f_{R}}}
$$

and $g_{Z}^{f} \equiv T_{f}^{3}-Q_{f} s_{\theta_{W}}^{2}$. Using these expressions and the expansion formalism in a $\chi^{2}$ analysis, we obtain $95 \%$ CL limits for the operator coefficients.

The left panel of figure 2 shows our results for fits to the coefficients $\bar{c}_{L L}^{(3) l}, \bar{c}_{T}, \bar{c}_{W}+\bar{c}_{B}$, together with the coefficient $\bar{c}_{R}^{e}$ that affects the leptonic observables $\left\{\Gamma_{Z}, \sigma_{\text {had }}^{0}, R_{e}^{0}, R_{\mu}^{0}, R_{\tau}^{0}\right.$, $\left.A_{\mathrm{FB}}^{0, e}, m_{W}\right\}$. The upper (green) bars indicate the ranges for each of the coefficients varied individually, assuming that the other coefficients vanish, and the lower (red) bars show the ranges for a global fit in which all the coefficients are varied simultaneously. In both fits, the coefficients are all quite compatible with zero, with ranges $\sim \pm 0.001$ in the singlecoefficient analysis, increasing in the global fit up to $\sim \pm 0.004$ for the coefficient $\bar{c}_{T}$ in the 

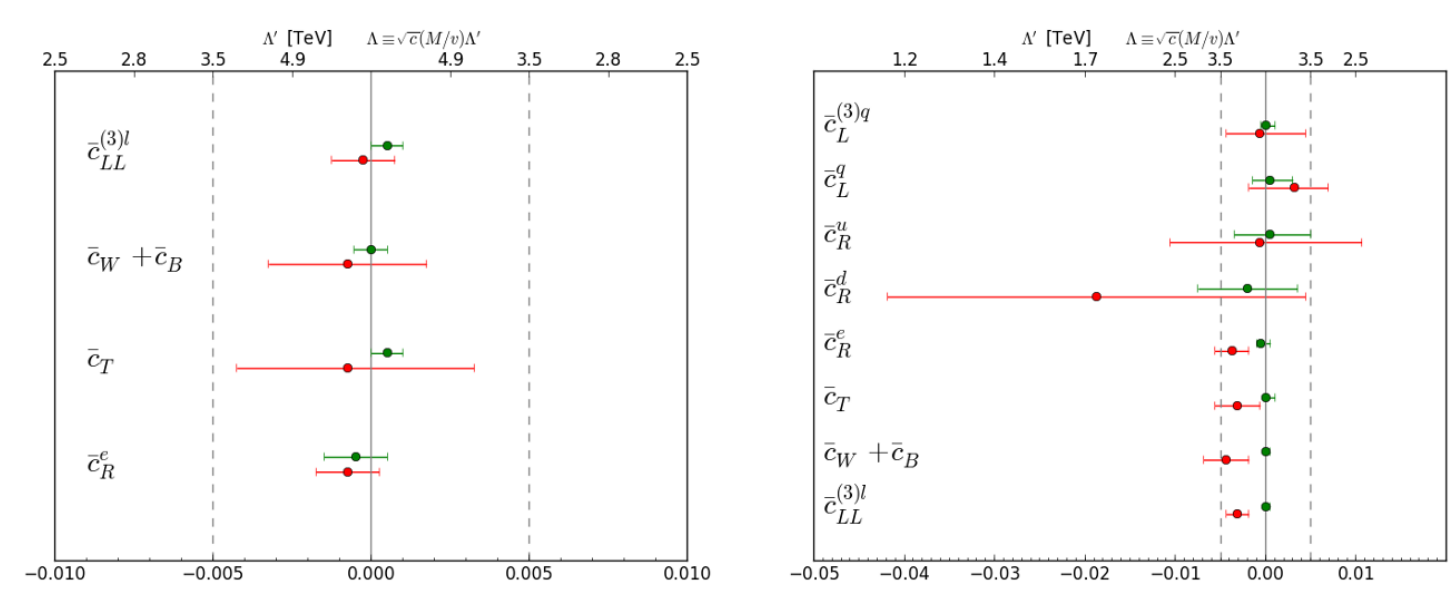

Figure 2. The 95\% CL ranges found in analyses of the leptonic observables (left panel) and including also the hadronic observables (right panel). In each case, the upper (green) bars denote single-coefficient fits, and the lower (red) bars denote multi-coefficient fits. The upper-axis should be read $\times \frac{m_{W}}{v} \sim 1 / 3$ for $\bar{c}_{W}+\bar{c}_{B}$.

multi-coefficient analysis. ${ }^{7}$ The legend at the top of the left panel of figure 2 translates the ranges of the coefficients into ranges of sensitivity to a large mass scale $\Lambda$. We see that all the sensitivities are in the multi- $\mathrm{TeV}$ range, including in the global analysis.

The right panel of figure 2 shows the effect of including the hadronic observables, $\left\{R_{b}^{0}, R_{c}^{0}, A_{\mathrm{FB}}^{0, b}, A_{\mathrm{FB}}^{0, c}, A_{b}, A_{c}\right\}$, and the coefficients that contribute directly to them, namely $\bar{c}_{L}^{q}, \bar{c}_{L}^{(3) q}, \bar{c}_{R}^{u}$ and $\bar{c}_{R}^{d}$. The ranges for the single-variable fits to $\bar{c}_{L L}^{(3) l}, \bar{c}_{T}, \bar{c}_{W}+\bar{c}_{B}$ and $\bar{c}_{R}^{e}$ (upper,green lines) are the same as in the left panel, but the horizontal scales are different, as seen immediately by comparing the separations of the vertical black dashed 'tramlines'. The ranges of these coefficients are altered significantly in the global 8-coefficient fit (lower, red lines) and we see significant tension with the null hypotheses for $\bar{c}_{L L}^{(3) l}, \bar{c}_{T}, \bar{c}_{W}+\bar{c}_{B}$ and $\bar{c}_{R}^{e}$, which reflects the well-known tension between the Standard Model and heavy-flavour measurements at the $Z$ peak. However, values of $\bar{c}_{L L}^{(3) l}, \bar{c}_{T}, \bar{c}_{W}+\bar{c}_{B}$ and $\bar{c}_{R}^{e}$ between 0 and -0.01 are favoured, corresponding to $\Lambda \gtrsim 2.5 \mathrm{TeV}$. The ranges of $\bar{c}_{L}^{q}, \bar{c}_{L}^{(3) q}, \bar{c}_{R}^{u}$ and $\bar{c}_{R}^{d}$ are considerably broader in both fits, particularly in the global 8-coefficient fit, most notably $\bar{c}_{R}^{u}$ and $\bar{c}_{R}^{d}$, with values of the latter approaching -0.05 being allowed at the $95 \% \mathrm{CL}$.

\section{Triple-gauge and Higgs couplings at the LHC}

In previous work [89] we used LHC measurements of Higgs signal strengths together with differential distributions in Higgs associated production measurements by ATLAS and D0 to constrain all the dimension- 6 operators affecting Higgs physics. The associated production information was vital in eliminating a blind direction, which can also be closed by including TGC measurements. These are most precisely measured by LEP, but it has been

\footnotetext{
${ }^{7}$ We note that larger marginalized ranges for $\bar{c}_{R}^{e}$ and $\bar{c}_{L L}^{(3) l}$ are found in [88], warranting further cross-checks.
} 


\begin{tabular}{|c|c|c|c|}
\hline Operator & \multirow{2}{*}{ Coefficient } & \multicolumn{2}{|c|}{ LHC Constraints } \\
& & Individual & Marginalized \\
\hline $\mathcal{O}_{W}=\frac{i g}{2}\left(H^{\dagger} \sigma^{a} \stackrel{\leftrightarrow}{D^{\mu}} H\right) D^{\nu} W_{\mu \nu}^{a}$ & $m_{W}^{2}$ & $(-0.022,0.004)$ & $(-0.035,0.005)$ \\
$\mathcal{O}_{B}=\frac{i g^{\prime}}{2}\left(H^{\dagger} \stackrel{\leftrightarrow}{D^{\mu}} H\right) \partial_{W}{ }^{\nu} B_{\mu \nu}$ & & & \\
\hline $\mathcal{O}_{H W}=i g\left(D^{\mu} H\right)^{\dagger} \sigma^{a}\left(D^{\nu} H\right) W_{\mu \nu}^{a}$ & $\frac{m_{W}^{2}}{\Lambda^{2}} c_{H W}$ & $(-0.042,0.008)$ & $(-0.035,0.015)$ \\
\hline $\mathcal{O}_{H B}=i g^{\prime}\left(D^{\mu} H\right)^{\dagger}\left(D^{\nu} H\right) B_{\mu \nu}$ & $\frac{m_{W}^{2}}{\Lambda^{2}} c_{H B}$ & $(-0.053,0.044)$ & $(-0.045,0.075)$ \\
\hline $\mathcal{O}_{3 W}=\frac{1}{3 !} g \epsilon_{a b c} W_{\mu}^{a} W_{\nu \rho}^{b} W^{c} \rho \mu$ & $\frac{m_{W}^{2}}{\Lambda^{2}} c_{3 W}$ & $(-0.083,0.045)$ & $(-0.083,0.045)$ \\
\hline $\mathcal{O}_{g}=g_{s}^{2}|H|^{2} G_{\mu \nu}^{A} G^{A \mu \nu}$ & $\frac{m_{W}^{2}}{\Lambda^{2}} c_{g}$ & $(0,3.0) \times 10^{-5}$ & $(-3.2,1.1) \times 10^{-4}$ \\
\hline $\mathcal{O}_{\gamma}=g^{\prime 2}|H|^{2} B_{\mu \nu} B^{\mu \nu}$ & $\frac{m_{W}^{2}}{\Lambda^{2}} c_{\gamma}$ & $(-4.0,2.3) \times 10^{-4}$ & $(-11,2.2) \times 10^{-4}$ \\
\hline $\mathcal{O}_{H}=\frac{1}{2}\left(\partial^{\mu}|H|^{2}\right)^{2}$ & $\frac{v^{2}}{\Lambda^{2}} c_{H}$ & $(-0.14,0.194)$ & $(-,-)$ \\
\hline $\mathcal{O}_{f}=y_{f}|H|^{2} \bar{F}_{L} H^{(c)} f_{R}+$ h.c. & $\frac{v^{2}}{\Lambda^{2}} c_{f}$ & $(-0.084,0.155)\left(c_{u}\right)$ & $(-,-)$ \\
& & $(-0.198,0.088)\left(c_{d}\right)$ & $(-,-)$ \\
\hline
\end{tabular}

Table 2. List of operators in our basis entering in LHC Higgs (including D0 associated production) and TGC physics, together with $95 \%$ CL bounds when individual coefficients are switched on one at a time, and marginalized in a simultaneous fit.

recently pointed out that the LEP TGC constraints $^{8}$ have a direction of limited sensitivity due to accidental partial cancellations [98]. Meanwhile, TGCs have been analysed at $8 \mathrm{TeV}$ at the LHC by both the CMS and ATLAS experiments [95-97], and here we study their potential to complement Higgs physics in constraining a complete set of dimension- 6 operators.

\subsection{TGC constraints on dimension- 6 operator coefficients}

The operators affecting Higgs physics and TGCs in the basis we adopt are listed in table 2, with the Lagrangian given by

$$
\begin{aligned}
\mathcal{L}_{\text {dim- } 6} \supset & \frac{\bar{c}_{W}}{m_{W}^{2}} \mathcal{O}_{W}+\frac{\bar{c}_{B}}{m_{W}^{2}} \mathcal{O}_{B}+\frac{\bar{c}_{H W}}{m_{W}^{2}} \mathcal{O}_{H W}+\frac{\bar{c}_{H B}}{m_{W}^{2}} \mathcal{O}_{H B}+\frac{\bar{c}_{\gamma}}{m_{W}^{2}} \mathcal{O}_{\gamma}+\frac{\bar{c}_{g}}{m_{W}^{2}} \mathcal{O}_{g} \\
& +\frac{\bar{c}_{3 W}}{m_{W}^{2}} \mathcal{O}_{3 W}+\sum_{f=t, b, \tau} \frac{\bar{c}_{f}}{v^{2}} \mathcal{O}_{f}+\frac{\bar{c}_{H}}{v^{2}} \mathcal{O}_{H}+\frac{\bar{c}_{6}}{v^{2}} \mathcal{O}_{6} .
\end{aligned}
$$

The constraint at the per-mille level on the combination $\bar{c}_{W}+\bar{c}_{B}$ obtained in the previous section allows us to set $\bar{c}_{B}=-\bar{c}_{W}$ (or equivalently to constrain the direction $\bar{c}_{W}-\bar{c}_{B}$ ). Ignoring the unconstrained operator $\mathcal{O}_{6}$ that affects the Higgs self-couplings and (for simplicity) setting $\bar{c}_{b}=\bar{c}_{\tau} \equiv \bar{c}_{d}$ then reduces the number of independent coefficients to nine. The coefficients $\bar{c}_{W}, \bar{c}_{H W}, \bar{c}_{H B}$ and $\bar{c}_{3 W}$ affect TGCs, with $\bar{c}_{3 W}$ being limited only by TGC measurements, since it does not affect Higgs physics.

\footnotetext{
${ }^{8}$ See also [119] for a recent discussion on the use of TGC observables as reported by LEP for constraining dimension-6 operators in different bases.
} 


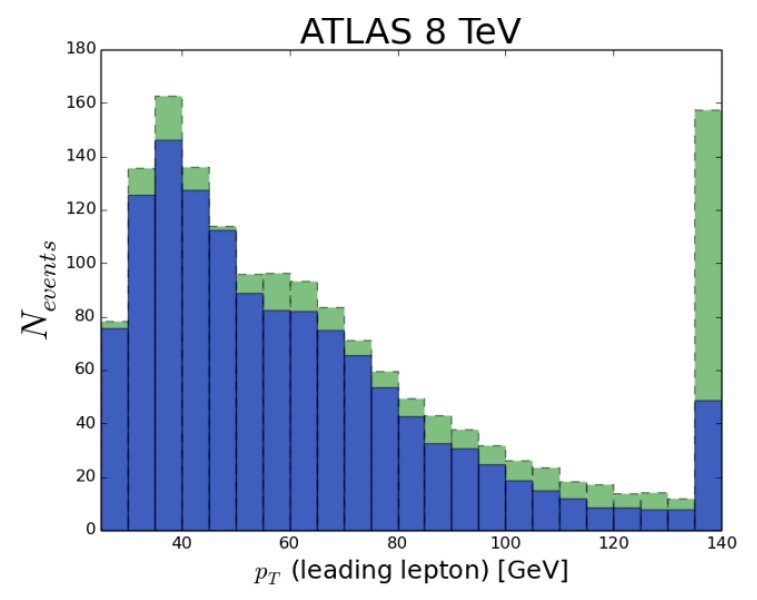

Figure 3. The same-flavour $p_{T}$ distribution of the leading lepton after the TGC analysis cuts for ATLAS at $8 \mathrm{TeV}$. The Standard Model distribution is shown in blue with solid lines, and the effect of $\bar{c}_{H W}=0.1$ is superimposed in green with dashed lines.

Note that our parametrization in terms of dimension-six effective operators are related to the anomalous coupling characterization [120-122], and the translation in written in the tables in ref. [118]. The kappa-formalism [123] can be linked to the EFT and AC characterization only at the level of total cross sections.

We calculate the TGCs in the presence of dimension- 6 operators using the FeynRules implementation of [118] in MadGraph v2.1.2 [124], interfaced with Pythia [125] and Delphes [126]. In the case of ATLAS, we implement the analysis given in [97]. This requires events that pass the selection cuts to have exactly 2 opposite-sign leptons with no jets, $p_{T}>$ $25(20) \mathrm{GeV}$ for leading (sub-leading) leptons, $m_{l l}>15(10) \mathrm{GeV}$ and $E_{T}^{\text {miss }}>45(15) \mathrm{GeV}$ for same-flavour (different-flavour) lepton pairs, as well as $\left|m_{l l}-m_{Z}\right|>15 \mathrm{GeV}$ for the same-flavour case. Similarly, following [95, 96], for the CMS cuts we require 2 oppositesign leptons with $p_{T}>20 \mathrm{GeV}$, total lepton $p_{T}>45 \mathrm{GeV}$ and $75 \mathrm{GeV}<m_{l l}<105 \mathrm{GeV}$, $E_{T}^{\text {miss }}>37(20) \mathrm{GeV}$ and $m_{l l}>20(12) \mathrm{GeV}$ for same-flavour (opposite-flavour) pairs, and no jets with $|\eta|<5, E_{T}>30 \mathrm{GeV}$.

The resulting $p_{T}$ distribution of the leading lepton for the ATLAS $8 \mathrm{TeV}$ analysis is shown in figure 3 including $\bar{c}_{H W}=0.1$ as well as the Standard Model contribution. ${ }^{9}$ We focus on the number of events in the last (overflow) bin, since this has the highest signalto-background ratio and grows rapidly as a function of this and the other dimension- 6 coefficients. ${ }^{10}$ We prefer to keep only the linear dependences on the dimension- 6 coefficients, considering that it is not consistent to keep terms that are quadratic in the dimension- 6 coefficients if one does not have reason to expect that the coefficients of dimension- 8 operators would be suppressed. As an example, we note that the signal-strength dependence of the overflow bin on $\bar{c}_{H W}$ for the ATLAS 8-TeV same-flavour distribution is found to be

$$
\mu_{\text {last-bin }}^{\mathrm{ATLAS} 8}=1+3.45 \bar{c}_{H W}+234 \bar{c}_{H W}^{2},
$$

\footnotetext{
${ }^{9}$ The applicability of the effective field theory approach to this TGC analysis is discussed in the appendix.

${ }^{10}$ The validity of the effective field theory at such high $p_{T}$ may be restricted only to certain models $[127$, 128], but the range of validity will increase as the current precision of LHC TGC measurements is improved.
} 

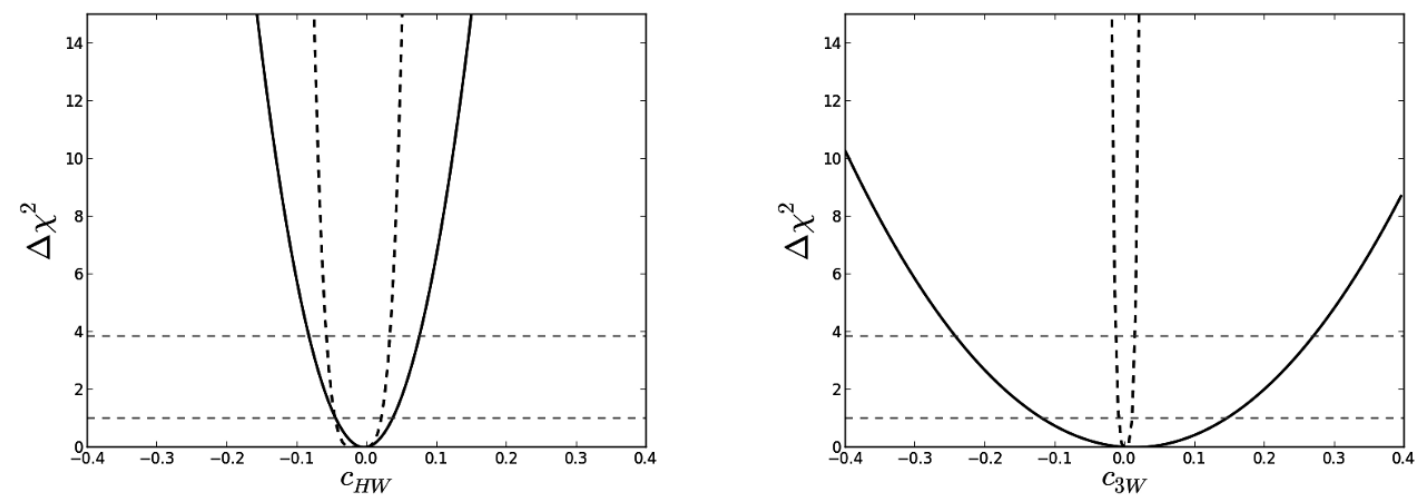

Figure 4. Comparisons between the $\chi^{2}$ functions from fits to the same-flavour ATLAS distribution including only linear (solid lines) and also quadratic (dashed lines) dependences on the dimension- 6 coefficients $\bar{c}_{H W}$ (left panel) and $\bar{c}_{3 W}$ (right panel).

and we keep only the linear term in our global fits. The constraints obtained using this linear (quadratic) dependence on the dimension- 6 coefficients are plotted as solid (dashed) lines in figure 4. The left panel is for $\bar{c}_{H W}$, and right panel is for $\bar{c}_{3 W}$. When deriving constraints we use the background and Standard Model signal Monte-Carlo (MC) distributions of the leading lepton $p_{T}$ provided by the experiments, and marginalize over the MC error. This is given along with the observed number of events and their errors in [97] for ATLAS and $[95,96]$ for CMS. We see that the quadratic and linear fits for $\bar{c}_{H W}$ are quite similar, whereas the constraint from the (preferred) linear fit for $\bar{c}_{3 W}$ is significantly weaker than that from the (deprecated) quadratic fit.

For the full global fit we use the same-flavour and different-flavour distributions for ATLAS at $8 \mathrm{TeV}$ and the CMS 7 and $8 \mathrm{TeV}$ data. In figure 5 we compare the constraints from the combination of the ATLAS and CMS TGC measurements with the LHC Higgs signal-strength data on each of the dimension-6 coefficients $\bar{c}_{W}, \bar{c}_{H W}$ and $\bar{c}_{H B}$ (top row), $\bar{c}_{g}, \bar{c}_{\gamma}$ and $\bar{c}_{3 W}$ (middle row), and $\bar{c}_{b}, \bar{c}_{t}$ and $\bar{c}_{H}$ (bottom row). ${ }^{11}$ The purple line represents the combination of LHC signal-strength constraints with the ATLAS 8-TeV TGC measurements, the blue line the combination of CMS 7- and 8-TeV constraints, and the red line uses all the sets of LHC TGC constraints. We use the signal-strength information on the $W^{+} W^{-(*)}, Z Z^{(*)}, \gamma \gamma, Z \gamma$, and $\tau^{+} \tau^{-}$final states, whose likelihoods are obtained as explained in [89]. We observe that the constraints on the coefficient $\bar{c}_{3 W}$, which only affects TGCs, is at the same level as some of the other coefficients whose operators also affect Higgs physics.

The results in figure 5 are summarised in the marginalised 95\% CL ranges displayed in figure 6. Again, the LHC signal-strength data are always included, in combination with the ATLAS 8-TeV data (purple bars), the CMS 7- and 8-TeV data (blue bars) and all the LHC TGC data (red bars). As already mentioned, the LHC TGC data enables a competitive model-independent bound on the coefficient $\bar{c}_{3 W}$.

\footnotetext{
${ }^{11}$ We note that the constraints on the last three operators are relatively weak, but include them for information.
} 

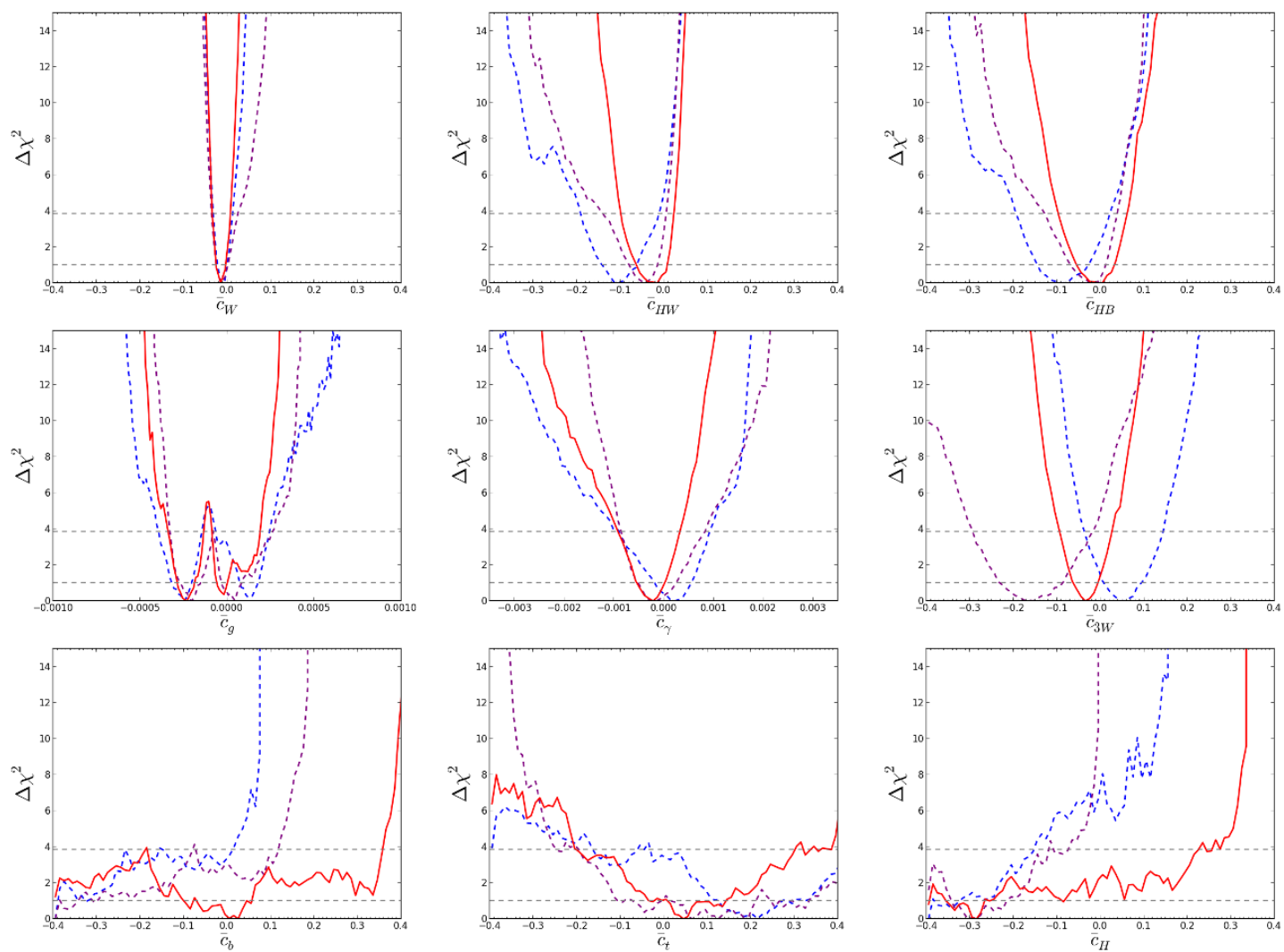

Figure 5. Comparisons of the constraints on the dimension-6 coefficients $\bar{c}_{W}, \bar{c}_{H W}$ and $\bar{c}_{H B}$ (top row), $\bar{c}_{g}, \bar{c}_{\gamma}$ and $\bar{c}_{3 W}$ (middle row), and $\bar{c}_{b}, \bar{c}_{t}$ and $\bar{c}_{H}$ (bottom row) provided by the LHC signal-strength data together with the ATLAS 8-TeV (purple lines), the CMS 7- and 8-TeV TGC measurements (blue lines) and their combination (red lines).

\subsection{Inclusion of Higgs associated production constraints}

We now include in our analysis the constraints from the kinematics of associated Higgs production, following the analysis of [89]. ${ }^{12}$ Figure 7 displays the marginalised $\chi^{2}$ distributions for each of the dimension- 6 coefficients $\bar{c}_{W}, \bar{c}_{H W}$ and $\bar{c}_{H B}$ (top row), $\bar{c}_{g}, \bar{c}_{\gamma}$ and $\bar{c}_{3 W}$ (middle row), and $\bar{c}_{b}, \bar{c}_{t}$ and $\bar{c}_{H}$ (bottom row). ${ }^{13}$ In each panel, the dashed blue line includes the Higgs signal strengths measured at the LHC and the constraints from the kinematic distributions for associated $H+V$ production measured by ATLAS and D0, whereas the solid red line includes the signal strengths and the LHC TGC measurements. The solid black lines include all the constraints: the signal strengths, the kinematic distributions and the TGCs measured at the LHC. We see that the LHC TGC measurements are the strongest for $\bar{c}_{W}$ and $\bar{c}_{3 W}$ : in particular, they are necessary to obtain any meaningful constraint on $\bar{c}_{3 W}$, which cannot be constrained at all by Higgs physics along as the marginalized

\footnotetext{
${ }^{12}$ The applicability of the effective field theory approach to this associated production analysis is discussed in the appendix.

${ }^{13}$ We note again that the constraints on the last three operators are relatively weak, but include them for information.
} 


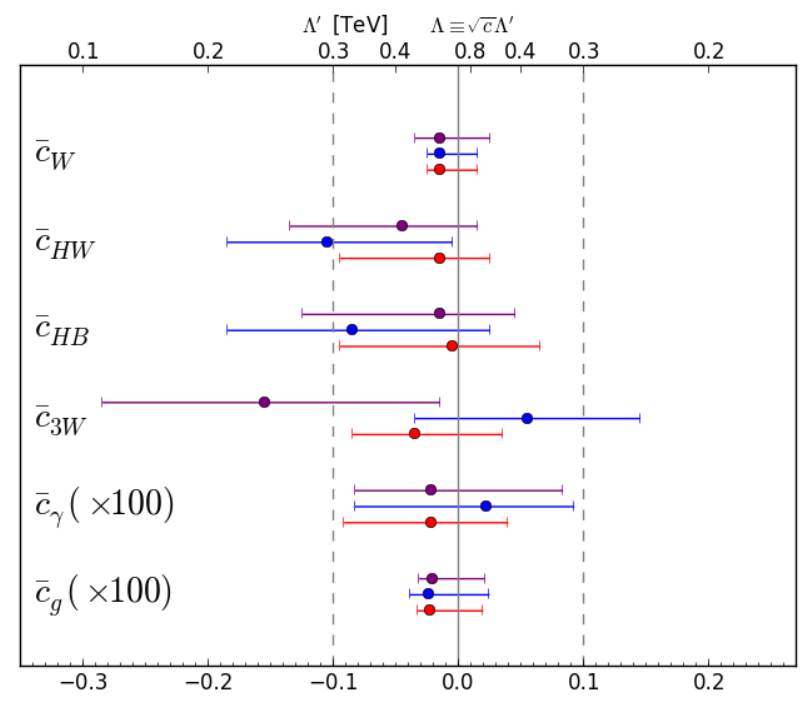

Figure 6. The marginalised $95 \% \mathrm{CL}$ ranges for the dimension- 6 operator coefficients obtained by combining the LHC signal-strength data with the ATLAS 8-TeV TGC data (purple bars), the CMS 7- and 8 -TeV TGC measurements (blue bars), and their combination (red bars). Note that $\bar{c}_{\gamma, g}$ are shown $\times 100$, so for these coefficients the upper axis should therefore be read $\times 10$.

likelihood (shown as a dashed blue line) fluctuates stochastically over a range larger than that displayed. On the other hand, the Higgs constraints are more important for $\bar{c}_{H W}$, $\bar{c}_{H B}$ and $\bar{c}_{g}$, whereas the TGC and Higgs constraints are of comparable importance for the other coefficients.

The results of our fits are summarised in figure 8. The individual 95\% CL constraints obtained by switching one coefficient on at a time are shown as green bars. The other lines are the marginalised 95\% ranges obtained using the LHC signal-strength data in combination with the kinematic distributions for associated $H+V$ production measured by ATLAS and D0 (blue bars), in combination with the LHC TGC data (red lines), and in combination with both the associated production and TGC data (black bars). We see again that the LHC TGC constraints are the most important for $\bar{c}_{W}$ and $\bar{c}_{3 W}$, whereas the Higgs constraints are more important for $\bar{c}_{H W}, \bar{c}_{H B}$ and $\bar{c}_{g}$. Our numerical results for the 95\% CL ranges for these coefficients are shown alongside the operator definitions in table 2. Results for the coefficients $c_{b}, c_{t}$ and $c_{H}$ are shown in the case of one-by-one constraints, but once other Higgs-gauge bosons are included in the global fit the sensitivities to them is reduced to current limits on $h \rightarrow b \bar{b}$ in associated production and $t \bar{t} h$.

\section{Application to the Two-Higgs Doublet Model}

We now discuss an example of the application of our constraints to a specific ultra-violet (UV) completion of the effective field theory. The case of a singlet scalar and stops contributing to dimension- 6 operators was recently considered in [129]. Here we briefly look at applying our constraints to the $2 \mathrm{HDM}$ scenario, which is worth further investigation [130]. 

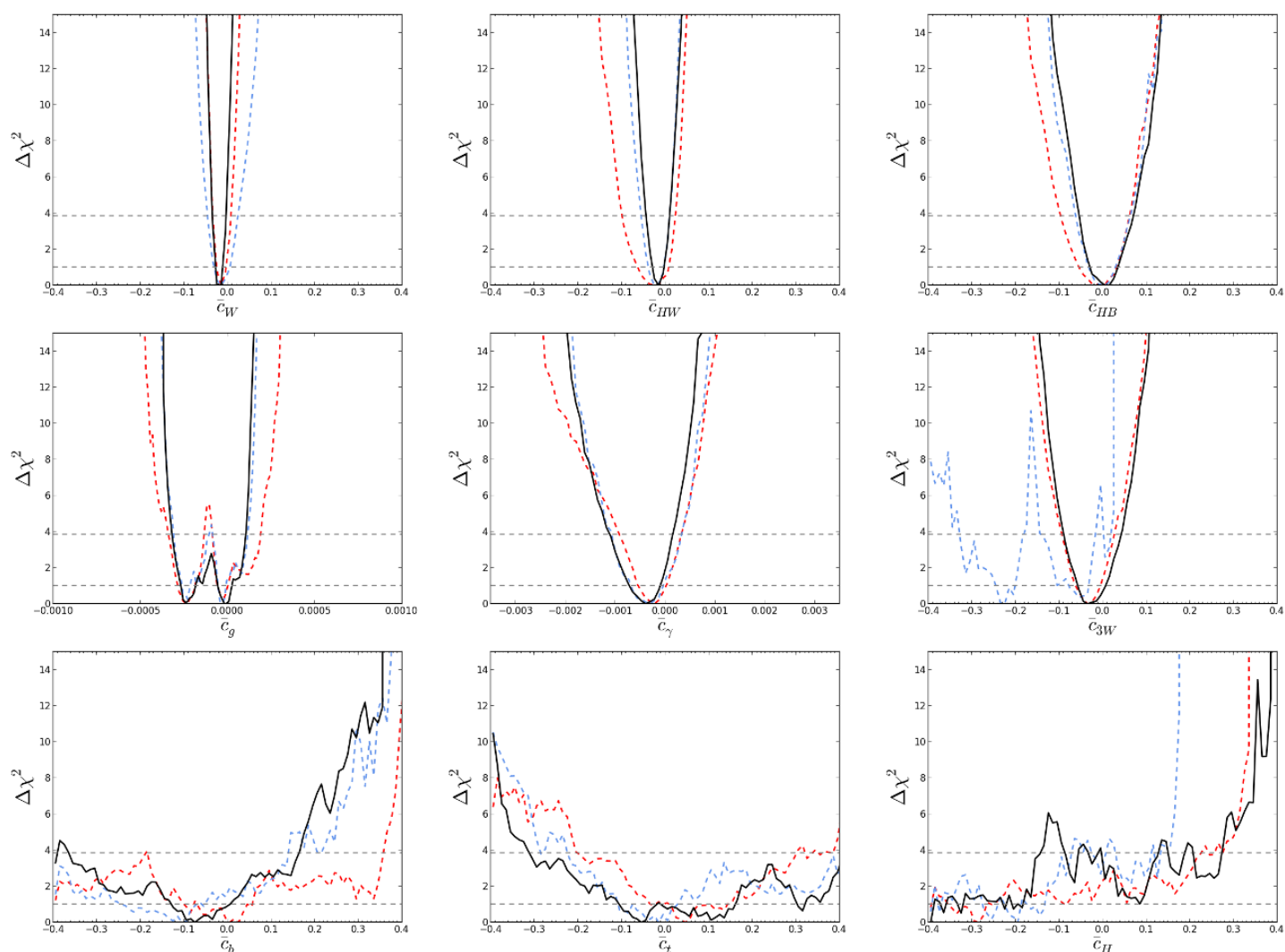

Figure 7. The marginalised $\chi^{2}$ distributions for each of the dimension- 6 coefficients $\bar{c}_{W}, \bar{c}_{H W}$ and $\bar{c}_{H B}$ (top row), $\bar{c}_{g}, \bar{c}_{\gamma}$ and $\bar{c}_{3 W}$ (middle row), and $\bar{c}_{b}, \bar{c}_{t}$ and $\bar{c}_{H}$ (bottom row), including the signal strengths measured at the LHC and the constraints from the kinematic distributions for associated $H+V$ production measured by ATLAS and D0 (dashed blue lines), the signal strengths and the LHC TGC measurements (red lines), and all the constraints (black lines).

We will be interested in particular in the case of the 2HDM in the alignment limit [131, 132], where the light Higgs couples to fermions and gauge bosons as the SM-Higgs, and all new effects are then through loops of the heavy scalars in the $2 \mathrm{HDM}$, as opposed to the usual limits coming from deviations of the Higgs couplings through mixing.

In a large range of models, including the $2 \mathrm{HDM}$ in this limit, the only coupling of the Higgs to massive vector bosons has the following Lorentz structure

$$
h W_{\mu \nu} W^{\mu \nu} .
$$

The translation between this Higgs anomalous coupling and the operators is given in [118] (see also [133]). The following constraints

$$
\bar{c}_{H W}=-\bar{c}_{W}, \bar{c}_{H B}=-\bar{c}_{B}
$$

are then satisfied at the UV scale. We recall from section 2 that, in addition, the EWPTs impose the constraint $\bar{c}_{W} \simeq-\bar{c}_{B}$, implying that, to a good approximation

$$
\bar{c}_{W}=-\bar{c}_{B}=-\bar{c}_{H W}=\bar{c}_{H B}
$$




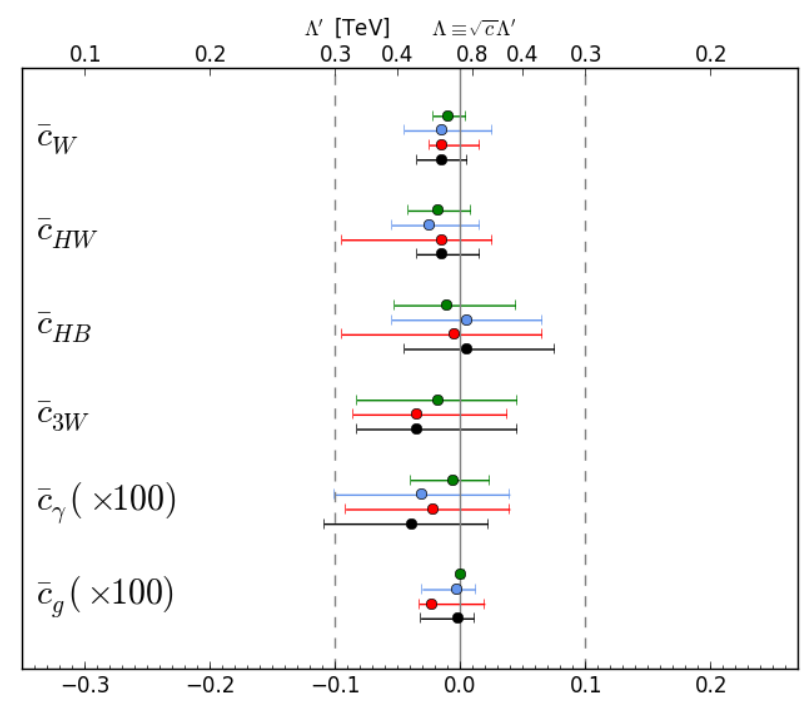

Figure 8. The 95\% CL constraints obtained for single-coefficient fits (green bars), and the marginalised $95 \%$ ranges for the LHC signal-strength data combined with the kinematic distributions for associated $H+V$ production measured by ATLAS and D0 (blue bars), combined with the LHC TGC data (red lines), and the global combination with both the associated production and TGC data (black bars). Note that $\bar{c}_{\gamma, g}$ are shown $\times 100$, so for these coefficients the upper axis should therefore be read $\times 10$.

with corrections due to renormalization-group running effects that are negligible compared to the precision of the current LHC constraints. Moreover, in the 2DHM one also finds generically that $\bar{c}_{3 W}$ is suppressed [130]

$$
\bar{c}_{3 W} \sim \mathcal{O}(0.1) g^{2} \bar{c}_{H W},
$$

so that it can be an order of magnitude smaller. In our application to the $2 \mathrm{HDM}$ we set it to zero, as well as using the constraints (4.3). Note that in this case, the fit to electroweak data would be complementary to the LHC constraints, as the same operators involved in the Higgs data would be affecting TGCs, LEP and LHC [88]. Below we give the results of the fit using LHC diboson and Higgs data only, as with the combination of diboson ATLAS and CMS data, the inclusion of LEP data does not substantially affect our results.

Examples of models in this class include a general two-Higgs doublet model (2HDM) [130], supersymmetry with electroweakino/sfermion loops [134], and the exchange of a radion/dilaton particle [133]. In the former two models these operators are generated at loop level, whereas in the third case the operators appear at tree-level through the exchange of the radion/dilaton particle. In the loop-induced cases, the validity of the effective theory is typically $\sqrt{\hat{s}} \sim 2 M$, where $M$ is the mass scale of the heavy states. In $2 \mathrm{HDMs}$ one would usually finds modifications of the coupling of the $\mathrm{H}$

Figure 9 shows the $\chi^{2}$ distributions we find in a global fit to the three independent dimension- 6 coefficients of the $2 \mathrm{HDM}, \bar{c}_{W}, \bar{c}_{g}$ and $\bar{c}_{\gamma}$ obtained under these assumptions. These distributions have been obtained including all the constraints from the signal strengths measured at the LHC, the constraints from the kinematic distributions for asso- 

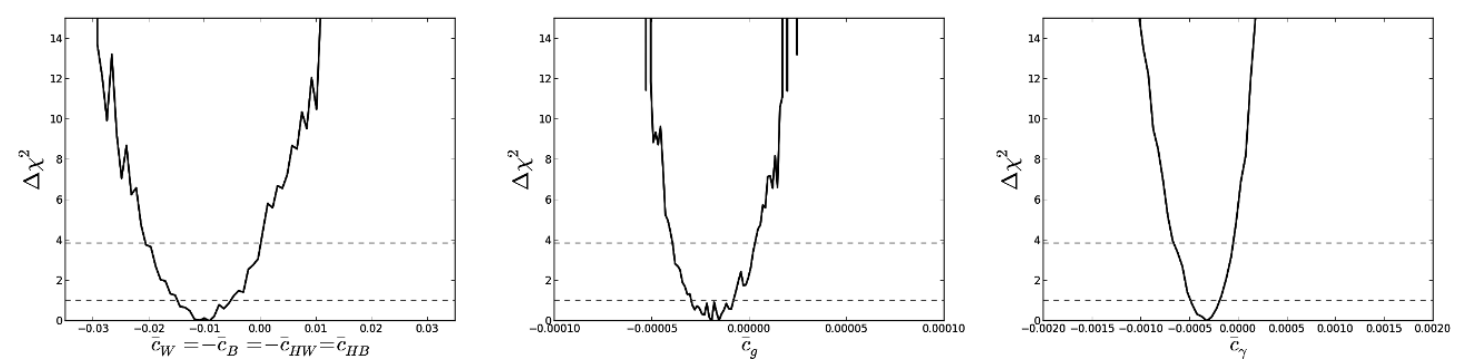

Figure 9. The marginalised $\chi^{2}$ distributions for the coefficients $\bar{c}_{W}=-\bar{c}_{B}=-\bar{c}_{H W}=\bar{c}_{H B}, \bar{c}_{g}$, and $\bar{c}_{\gamma}$ of the three independent dimension- 6 operators in the $2 \mathrm{HDM}$ under the assumptions stated in the text.

ciated $H+V$ production measured by ATLAS and D0, and the LHC TGC measurements. We find the following $95 \%$ CL ranges

$$
\begin{aligned}
\bar{c}_{W} & \in-(0.02,0.0004) \\
\bar{c}_{g} & \in-(0.00004,0.000003) \\
\bar{c}_{\gamma} & \in-(0.0006,-0.00003)
\end{aligned}
$$

in this particular class of models. The translation between the coefficients and the 2HDM will be presented in ref. [130], but let us comment here how these values relate to the validity of the effective theory. Roughly speaking, we find that $\bar{c} \simeq \frac{\lambda}{192 \pi^{2}} \frac{v^{2}}{M^{2}}$, with $\lambda$ a quartic coupling in the $2 \mathrm{HDM}$ scalar potential and $M$ the mass of the heavy particles. Hence, a limit of the order of $10^{-4}$ would lead to a mass limit of $2 \mathrm{TeV}$ for $\lambda=4 \pi$, and decrease as the the coupling becomes smaller.

\section{Conclusions}

The main lesson learned from Run I of the LHC is that, to a first approximation, we seem to have a Standard Model-like Higgs sector. Taken together with the fact that there is currently no clear evidence for any new physics beyond the Standard Model, it is natural to consider the Standard Model in its complete effective theory formulation. Such a (relatively) model-independent framework parameterises all the possible ways in which decoupled new physics may affect measurements at different experiments in a correlated and motivated way.

We have analysed in this paper the constraints imposed on the coefficients of dimension6 operator extensions of the Standard Model by EWPTs and LHC data. We first analysed the EWPTs using the expansion formalism of [106], which is particularly appropriate for models where the dominant corrections to the Standard Model predictions are not necessarily present only in the vector-boson self-energies, as is the case for general dimension- 6 extensions of the Standard Model. We confirm previous findings that the EWPTs provide particularly important constraints on some of the operator coefficients, as shown in figure 2 and table 1 . 
We then analysed the TGC data now available from ATLAS at $8 \mathrm{TeV}$ and from CMS at 7 and $8 \mathrm{TeV}$. We find that the most important aspects of the data are the highestenergy (overflow) bins in the lepton $p_{T}$ distributions, as illustrated in figure 3 , and use these together with Higgs signal strength measurements to obtain constraints on a set of nine operator coefficients, as shown in figures 5 and 6 . We then combined these LHC TGC constraints with the constraints provided by measurements of the kinematics of Higgs production in association with massive vector bosons at the Tevatron and the LHC, obtaining the results shown in figures 7 and 8 and table 2. As seen there, we find that completing the Higgs signal strengths constraints on dimension- 6 operators using the LHC TGCs provide the strongest LHC constraints on some of the coefficients, whereas the Higgs differential distributions in associated production are more important for some others, with both making important contributions in some cases. In particular, we obtain the first bounds on the coefficient $\bar{c}_{3 W}$ for a complete basis in the effective Standard Model. It is only by combining the TGC and Higgs constraints that one can obtain a complete picture of the possible ranges of the dimension-6 operator coefficients after LHC Run 1.

It is to be expected that Run 2 of the LHC will provide important improvements in the sensitivity of LHC probes of possible dimension- 6 operators. These improvements will come not only from the greater statistics, but also from the greater kinematic range that will strengthen the power of the associated Higgs production kinematics and the TGC constraints, in particular. At the moment we know that the Standard Model is very effective: LHC Run 2 data will give us a better idea just how effective it is, and perhaps provide some pointers to the nature of the new physics that surely lies beyond it at higher energies.

\section{Acknowledgments}

We thank Francesco Riva for useful conversations and Maxime Gouzevitch and Alexander Savin for helpful information about the CMS TGC distributions. The work of JE was supported partly by the London Centre for Terauniverse Studies (LCTS), using funding from the European Research Council via the Advanced Investigator Grant 26732, and partly by the STFC Grant ST/J002798/1. The work of VS was supported by the STFC Grant ST/J000477/1. The work of TY was supported by a Graduate Teaching Assistantship from King's College London.

\section{A Kinematics and the validity of the effective field theory}

We use in section 3 triple-gauge couplings and information on kinematic distributions in Higgs production in association with a vector boson production constraints, finding that typical $95 \% \mathrm{CL}$ constraints on the dimension- 6 coefficients are $\mathcal{O}\left(10^{-1}-10^{-2}\right)$. For example, for the operator $\bar{c}_{W}$ our limits are

$$
\bar{c}_{W} \in(-0.022,0.004) \text { [one-by-one] and }(-0.035,0.005) \text { [global] } .
$$



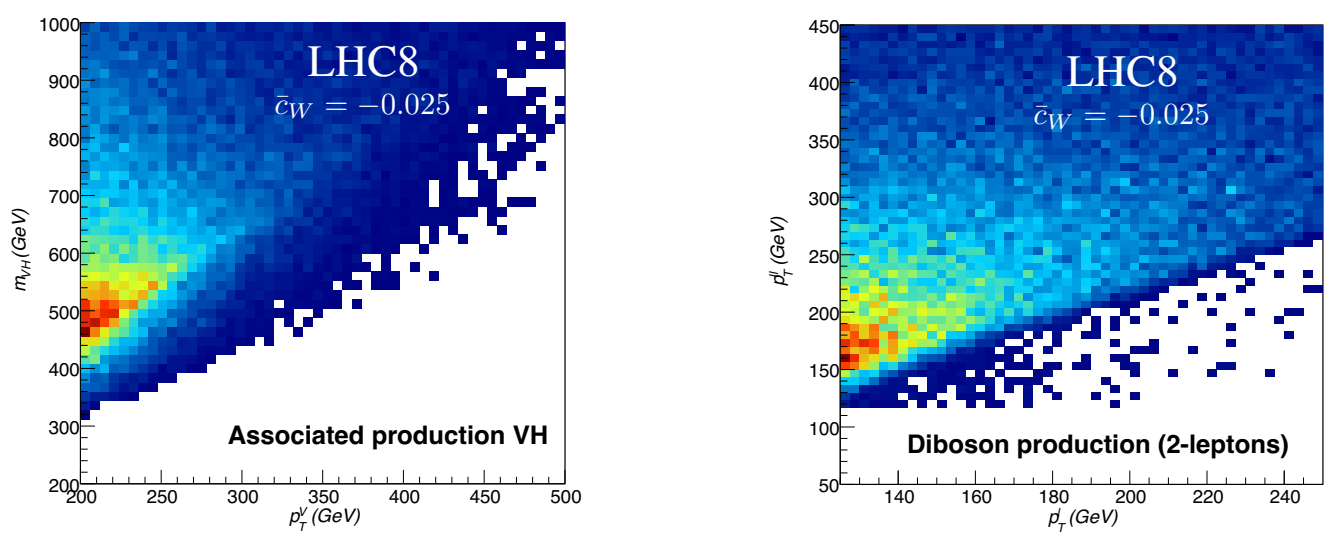

Figure 10. (Left) The kinematic distribution in the vector boson $p_{T}^{V}$ vs $m_{V H}$ plane for associated Higgs production at the LHC that would by induced by $\bar{c}_{W}=-0.025$. (Right) The kinematic distribution in the leading lepton $p_{T}$ vs $p_{T}^{\ell \ell}$ plane for diboson production at the LHC that would by induced by $\bar{c}_{W}=-0.025$.

Recalling the definition of the barred coefficients in eq. (2.6), one can interpret these limits in terms of new physics at scale $\Lambda$ coupled to the SM with strength $g_{\mathrm{NP}}$,

$$
\frac{\bar{c}_{W}}{m_{W}^{2}}=\frac{g_{\mathrm{NP}}^{2}}{\Lambda^{2}},
$$

upto a factor $g$ from the conventional definition of $\mathcal{O}_{W}$. The value of $\Lambda$ corresponding to a value of $\bar{c}_{W}$ can be read off the upper x-axis in figure 8 assuming $g_{\mathrm{NP}}^{2}=1$, where we see that the marginalized range for $\bar{c}_{W}$ corresponds to $\Lambda \sim 400-800 \mathrm{GeV}$. However $g_{\mathrm{NP}}$ may vary to be less than 1 in weakly-coupled scenarios, in which case the new physics scale is lowered, or up to $4 \pi$ for strongly-coupled new physics, which raises $\Lambda$. In general we have

$$
\Lambda_{\bar{c}_{W}} \simeq\left(\frac{g_{\mathrm{NP}}}{4 \pi}\right) 10 \mathrm{TeV}
$$

The question can be asked whether the effective Standard Model approach is justified.

In this appendix we address this question by considering the region where the most sensitivity is obtained, i.e., the last bin. First of all, it is important to note that the last bin is an overflow bin, containing all the events with $p_{T}$ above a specified cut. For example, in the TGC analysis shown in figure 3 the last bin corresponds to $p_{T}>135 \mathrm{GeV}$.

For a given value of $\Lambda$, one expects the effective theory to break down at parton energies $\sqrt{\hat{s}} \simeq \Lambda$, namely $m_{V V}$ and $m_{V H}$ in the diboson and VH production respectively. To illustrate this point, in figure 10 we show the kinematic distribution that would be induced by $\bar{c}_{W}=-0.025$ (our most conservative limit in $\bar{c}_{W}$ ) in the plane defined by the transverse momentum of the vector boson, $p_{T}^{V}$, and the invariant mass, $m_{V H}$, for associated Higgs production at the LHC in the 2-lepton channel. This plot corresponds to the last bin of the distribution, which has a cut $p_{T}^{V}>200 \mathrm{GeV}$. We see that in this bin typically $p_{T}^{V} \lesssim 250 \mathrm{GeV}$, i.e., there is not a large spread of events at large values of the distribution, and $\sqrt{\hat{s}}=m_{V H} \lesssim 550 \mathrm{GeV}$. 
One can perform a similar analysis in the di-boson production case. For comparison, we show in the right panel of figure 10 the $p_{T}$ distribution of the leading lepton in the $p p \rightarrow W^{+} W^{-} \rightarrow 2 \ell+\mathbb{E}_{T}$ production at LHC8 versus the transverse mass distribution of the two vector bosons, $p_{T}^{l l}$. For comparison with figure 3 , we infer that the overflow bin of $p_{T}>135 \mathrm{GeV}$ extends to about $160 \mathrm{GeV}$, and is correlated with $p_{T} \ell \ell<250 \mathrm{GeV}$.

Thus, in both the associated production and TGC cases, for $g_{\mathrm{NP}}=\mathcal{O}(1)$, equation (A.3) reassures us that the most important regions of the kinematical distributions are well within the ranges where one may expect the effective field theory to be a good enough approximation for our purposes.

Open Access. This article is distributed under the terms of the Creative Commons Attribution License (CC-BY 4.0), which permits any use, distribution and reproduction in any medium, provided the original author(s) and source are credited.

\section{References}

[1] ATLAS collaboration, Observation of a new particle in the search for the Standard Model Higgs boson with the ATLAS detector at the LHC, Phys. Lett. B 716 (2012) 1 [arXiv: 1207.7214] [INSPIRE].

[2] CMS collaboration, Observation of a new boson at a mass of $125 \mathrm{GeV}$ with the CMS experiment at the LHC, Phys. Lett. B 716 (2012) 30 [arXiv:1207.7235] [INSPIRE].

[3] M. Baak et al., Updated Status of the Global Electroweak Fit and Constraints on New Physics, Eur. Phys. J. C 72 (2012) 2003 [arXiv:1107.0975] [InSPIRE].

[4] D. Carmi, A. Falkowski, E. Kuflik and T. Volansky, Interpreting LHC Higgs Results from Natural New Physics Perspective, JHEP 07 (2012) 136 [arXiv:1202.3144] [INSPIRE].

[5] A. Azatov, R. Contino and J. Galloway, Model-Independent Bounds on a Light Higgs, JHEP 04 (2012) 127 [Erratum ibid. 1304 (2013) 140] [arXiv: 1202.3415] [inSPIRE].

[6] J.R. Espinosa, C. Grojean, M. Muhlleitner and M. Trott, Fingerprinting Higgs Suspects at the LHC, JHEP 05 (2012) 097 [arXiv: 1202.3697] [INSPIRE].

[7] P.P. Giardino, K. Kannike, M. Raidal and A. Strumia, Reconstructing Higgs boson properties from the LHC and Tevatron data, JHEP 06 (2012) 117 [arXiv:1203.4254] [INSPIRE].

[8] T. Li, X. Wan, Y.-k. Wang and S.-h. Zhu, Constraints on the Universal Varying Yukawa Couplings: from SM-like to Fermiophobic, JHEP 09 (2012) 086 [arXiv:1203.5083] [INSPIRE].

[9] M. Rauch, Determination of Higgs-boson couplings (SFitter), arXiv:1203.6826 [INSPIRE].

[10] J. Ellis and T. You, Global Analysis of Experimental Constraints on a Possible Higgs-Like Particle with Mass $125 \mathrm{GeV}$, JHEP 06 (2012) 140 [arXiv:1204.0464] [INSPIRE].

[11] A. Azatov, R. Contino, D. Del Re, J. Galloway, M. Grassi and S. Rahatlou, Determining Higgs couplings with a model-independent analysis of $h \rightarrow \gamma \gamma$, JHEP 06 (2012) 134 [arXiv: 1204.4817] [INSPIRE].

[12] M. Klute, R. Lafaye, T. Plehn, M. Rauch and D. Zerwas, Measuring Higgs Couplings from LHC Data, Phys. Rev. Lett. 109 (2012) 101801 [arXiv:1205.2699] [INSPIRE]. 
[13] L. Wang and X.-F. Han, The recent Higgs boson data and Higgs triplet model with vector-like quark, Phys. Rev. D 86 (2012) 095007 [arXiv: 1206.1673] [INSPIRE].

[14] D. Carmi, A. Falkowski, E. Kuflik and T. Volansky, Interpreting the $125 \mathrm{GeV}$ Higgs, Nuovo Cim. C 035 (2012) 315 [arXiv: 1206.4201] [INSPIRE].

[15] M.J. Dolan, C. Englert and M. Spannowsky, Higgs self-coupling measurements at the LHC, JHEP 10 (2012) 112 [arXiv:1206.5001] [INSPIRE].

[16] J. Chang, K. Cheung, P.-Y. Tseng and T.-C. Yuan, Distinguishing Various Models of the $125 \mathrm{GeV}$ Boson in Vector Boson Fusion, JHEP 12 (2012) 058 [arXiv: 1206.5853] [INSPIRE].

[17] S. Chang, C.A. Newby, N. Raj and C. Wanotayaroj, Revisiting Theories with Enhanced Higgs Couplings to Weak Gauge Bosons, Phys. Rev. D 86 (2012) 095015 [arXiv:1207.0493] [INSPIRE].

[18] I. Low, J. Lykken and G. Shaughnessy, Have We Observed the Higgs (Imposter)?, Phys. Rev. D 86 (2012) 093012 [arXiv:1207.1093] [INSPIRE].

[19] J. Ellis and T. You, Global Analysis of the Higgs Candidate with Mass 125 GeV, JHEP 09 (2012) 123 [arXiv:1207.1693] [INSPIRE].

[20] M. Montull and F. Riva, Higgs discovery: the beginning or the end of natural EWSB?, JHEP 11 (2012) 018 [arXiv:1207.1716] [INSPIRE].

[21] J.R. Espinosa, C. Grojean, M. Muhlleitner and M. Trott, First Glimpses at Higgs' face, JHEP 12 (2012) 045 [arXiv:1207.1717] [INSPIRE].

[22] D. Carmi, A. Falkowski, E. Kuflik, T. Volansky and J. Zupan, Higgs After the Discovery: A Status Report, JHEP 10 (2012) 196 [arXiv:1207.1718] [INSPIRE].

[23] S. Banerjee, S. Mukhopadhyay and B. Mukhopadhyaya, New Higgs interactions and recent data from the LHC and the Tevatron, JHEP 10 (2012) 062 [arXiv:1207.3588] [INSPIRE].

[24] F. Bonnet, T. Ota, M. Rauch and W. Winter, Interpretation of precision tests in the Higgs sector in terms of physics beyond the Standard Model, Phys. Rev. D 86 (2012) 093014 [arXiv: 1207.4599] [INSPIRE].

[25] T. Plehn and M. Rauch, Higgs Couplings after the Discovery, Europhys. Lett. 100 (2012) 11002 [arXiv: 1207.6108] [INSPIRE].

[26] A. Djouadi, Precision Higgs coupling measurements at the LHC through ratios of production cross sections, Eur. Phys. J. C 73 (2013) 2498 [arXiv:1208.3436] [INSPIRE].

[27] B. Batell, S. Gori and L.-T. Wang, Higgs Couplings and Precision Electroweak Data, JHEP 01 (2013) 139 [arXiv: 1209.6382] [INSPIRE].

[28] G. Moreau, Constraining extra-fermion(s) from the Higgs boson data, Phys. Rev. D 87 (2013) 015027 [arXiv: 1210.3977] [INSPIRE].

[29] G. Cacciapaglia, A. Deandrea, G.D. La Rochelle and J.-B. Flament, Higgs couplings beyond the Standard Model, JHEP 03 (2013) 029 [arXiv:1210.8120] [INSPIRE].

[30] E. Massó and V. Sanz, Limits on anomalous couplings of the Higgs boson to electroweak gauge bosons from LEP and the LHC, Phys. Rev. D 87 (2013) 033001 [arXiv:1211.1320] [INSPIRE].

[31] R.T. D'Agnolo, E. Kuflik and M. Zanetti, Fitting the Higgs to Natural SUSY, JHEP 03 (2013) 043 [arXiv:1212.1165] [INSPIRE]. 
[32] A. Azatov and J. Galloway, Electroweak Symmetry Breaking and the Higgs Boson: Confronting Theories at Colliders, Int. J. Mod. Phys. A 28 (2013) 1330004 [arXiv: 1212.1380] [INSPIRE].

[33] G. Bhattacharyya, D. Das and P.B. Pal, Modified Higgs couplings and unitarity violation, Phys. Rev. D 87 (2013) 011702 [arXiv:1212.4651] [InSPIRE].

[34] D. Choudhury, R. Islam and A. Kundu, Anomalous Higgs Couplings as a Window to New Physics, Phys. Rev. D 88 (2013) 013014 [arXiv:1212.4652] [INSPIRE].

[35] R.S. Gupta, M. Montull and F. Riva, SUSY Faces its Higgs Couplings, JHEP 04 (2013) 132 [arXiv: 1212.5240] [INSPIRE].

[36] G. Bélanger, B. Dumont, U. Ellwanger, J.F. Gunion and S. Kraml, Higgs Couplings at the End of 2012, JHEP 02 (2013) 053 [arXiv:1212.5244] [INSPIRE].

[37] K. Cheung, J.S. Lee and P.-Y. Tseng, Higgs Precision (Higgcision) Era begins, JHEP 05 (2013) 134 [arXiv: 1302.3794] [INSPIRE].

[38] A. Falkowski, F. Riva and A. Urbano, Higgs at last, JHEP 11 (2013) 111 [arXiv:1303.1812] [INSPIRE].

[39] P.P. Giardino, K. Kannike, I. Masina, M. Raidal and A. Strumia, The universal Higgs fit, JHEP 05 (2014) 046 [arXiv: 1303.3570] [INSPIRE].

[40] J. Ellis and T. You, Updated Global Analysis of Higgs Couplings, JHEP 06 (2013) 103 [arXiv: 1303.3879] [INSPIRE].

[41] J. Bernon, B. Dumont and S. Kraml, Status of Higgs couplings after run 1 of the LHC, Phys. Rev. D 90 (2014) 071301 [arXiv:1409.1588] [INSPIRE].

[42] T. Appelquist and J. Carazzone, Infrared Singularities and Massive Fields, Phys. Rev. D 11 (1975) 2856 [INSPIRE].

[43] W. Buchmüller and D. Wyler, Effective Lagrangian Analysis of New Interactions and Flavor Conservation, Nucl. Phys. B 268 (1986) 621 [inSPIRE].

[44] H.D. Politzer, Power Corrections at Short Distances, Nucl. Phys. B 172 (1980) 349 [INSPIRE].

[45] H. Kluberg-Stern and J.B. Zuber, Renormalization of Nonabelian Gauge Theories in a Background Field Gauge. 2. Gauge Invariant Operators, Phys. Rev. D 12 (1975) 3159 [INSPIRE].

[46] C. Grosse-Knetter, Effective Lagrangians with higher derivatives and equations of motion, Phys. Rev. D 49 (1994) 6709 [hep-ph/9306321] [InSPIRE].

[47] C. Arzt, Reduced effective Lagrangians, Phys. Lett. B 342 (1995) 189 [hep-ph/9304230] [INSPIRE].

[48] H. Simma, Equations of motion for effective Lagrangians and penguins in rare B decays, Z. Phys. C 61 (1994) 67 [hep-ph/9307274] [INSPIRE].

[49] J. Wudka, Electroweak effective Lagrangians, Int. J. Mod. Phys. A 9 (1994) 2301 [hep-ph/9406205] [INSPIRE].

[50] B. Grzadkowski, M. Iskrzynski, M. Misiak and J. Rosiek, Dimension-Six Terms in the Standard Model Lagrangian, JHEP 10 (2010) 085 [arXiv: 1008.4884] [INSPIRE]. 
[51] K. Hagiwara, S. Ishihara, R. Szalapski and D. Zeppenfeld, Low-energy effects of new interactions in the electroweak boson sector, Phys. Rev. D 48 (1993) 2182 [InSPIRE].

[52] K. Hagiwara, R. Szalapski and D. Zeppenfeld, Anomalous Higgs boson production and decay, Phys. Lett. B 318 (1993) 155 [hep-ph/9308347] [INSPIRE].

[53] F. Bonnet, M.B. Gavela, T. Ota and W. Winter, Anomalous Higgs couplings at the LHC and their theoretical interpretation, Phys. Rev. D 85 (2012) 035016 [arXiv:1105.5140] [INSPIRE].

[54] T. Corbett, O.J.P. Eboli, J. Gonzalez-Fraile and M.C. Gonzalez-Garcia, Constraining anomalous Higgs interactions, Phys. Rev. D 86 (2012) 075013 [arXiv:1207.1344] [INSPIRE].

[55] R. Contino, M. Ghezzi, C. Grojean, M. Muhlleitner and M. Spira, Effective Lagrangian for a light Higgs-like scalar, JHEP 07 (2013) 035 [arXiv: 1303.3876] [INSPIRE].

[56] W.-F. Chang, W.-P. Pan and F. Xu, Effective gauge-Higgs operators analysis of new physics associated with the Higgs boson, Phys. Rev. D 88 (2013) 033004 [arXiv:1303.7035] [inSPIRE].

[57] T. Corbett, O.J.P. Éboli, J. Gonzalez-Fraile and M.C. Gonzalez-Garcia, Determining Triple Gauge Boson Couplings from Higgs Data, Phys. Rev. Lett. 111 (2013) 011801 [arXiv: 1304.1151] [INSPIRE].

[58] A. Hayreter and G. Valencia, Constraints on anomalous color dipole operators from Higgs boson production at the LHC, Phys. Rev. D 88 (2013) 034033 [arXiv: 1304.6976] [INSPIRE].

[59] H. Mebane, N. Greiner, C. Zhang and S. Willenbrock, Constraints on Electroweak Effective Operators at One Loop, Phys. Rev. D 88 (2013) 015028 [arXiv:1306.3380] [InSPIRE].

[60] M.B. Einhorn and J. Wudka, The Bases of Effective Field Theories, Nucl. Phys. B 876 (2013) 556 [arXiv: 1307.0478] [inSPIRE].

[61] J. Elias-Miro, J.R. Espinosa, E. Masso and A. Pomarol, Higgs windows to new physics through $D=6$ operators: constraints and one-loop anomalous dimensions, JHEP 11 (2013) 066 [arXiv: 1308.1879] [INSPIRE].

[62] S. Banerjee, S. Mukhopadhyay and B. Mukhopadhyaya, Higher dimensional operators and the LHC Higgs data: The role of modified kinematics, Phys. Rev. D 89 (2014) 053010 [arXiv: 1308.4860] [INSPIRE].

[63] E. Boos, V. Bunichev, M. Dubinin and Y. Kurihara, Higgs boson signal at complete tree level in the SM extension by dimension-six operators, Phys. Rev. D 89 (2014) 035001 [arXiv: 1309.5410] [INSPIRE].

[64] B. Gripaios and D. Sutherland, Searches for CP-violating dimension-6 electroweak gauge boson operators, Phys. Rev. D 89 (2014) 076004 [arXiv:1309.7822] [INSPIRE].

[65] C.-Y. Chen, S. Dawson and C. Zhang, Electroweak Effective Operators and Higgs Physics, Phys. Rev. D 89 (2014) 015016 [arXiv:1311.3107] [INSPIRE].

[66] M. Dahiya, S. Dutta and R. Islam, Unitarizing VV Scattering in Light Higgs Scenarios, arXiv: 1311.4523 [INSPIRE].

[67] C. Grojean, E. Salvioni, M. Schlaffer and A. Weiler, Very boosted Higgs in gluon fusion, JHEP 05 (2014) 022 [arXiv: 1312.3317] [INSPIRE].

[68] J. Bramante, A. Delgado and A. Martin, Cornering a hyper Higgs boson: Angular kinematics for boosted Higgs bosons with top pairs, Phys. Rev. D 89 (2014) 093006 [arXiv:1402.5985] [INSPIRE]. 
[69] J.S. Gainer, J. Lykken, K.T. Matchev, S. Mrenna and M. Park, Beyond Geolocating: Constraining Higher Dimensional Operators in $H \rightarrow 4 \ell$ with Off-Shell Production and More, Phys. Rev. D 91 (2015) 035011 [arXiv:1403.4951] [INSPIRE].

[70] S. Bar-Shalom, A. Soni and J. Wudka, EFT naturalness: an effective field theory analysis of Higgs naturalness, arXiv:1405.2924 [INSPIRE].

[71] G. Amar et al., Exploration of the tensor structure of the Higgs boson coupling to weak bosons in $e^{+} e^{-}$collisions, JHEP 02 (2015) 128 [arXiv:1405.3957] [INSPIRE].

[72] A. Azatov, C. Grojean, A. Paul and E. Salvioni, Taming the off-shell Higgs boson, Zh. Eksp. Teor. Fiz. 147 (2015) 410 [arXiv:1406.6338] [INSPIRE].

[73] E. Masso, An Effective Guide to Beyond the Standard Model Physics, JHEP 10 (2014) 128 [arXiv: 1406.6376] [INSPIRE].

[74] R. Alonso, E.E. Jenkins and A.V. Manohar, Holomorphy without Supersymmetry in the Standard Model Effective Field Theory, Phys. Lett. B 739 (2014) 95 [arXiv:1409.0868] [INSPIRE].

[75] R.M. Godbole, D.J. Miller, K.A. Mohan and C.D. White, Jet substructure and probes of CP-violation in Vh production, arXiv:1409.5449 [INSPIRE].

[76] F. Goertz, A. Papaefstathiou, L.L. Yang and J. Zurita, Higgs boson pair production in the $D=6$ extension of the $S M$, arXiv:1410.3471 [INSPIRE].

[77] L. Lehman, Extending the Standard Model Effective Field Theory with the Complete Set of Dimension-7 Operators, Phys. Rev. D 90 (2014) 125023 [arXiv:1410.4193] [INSPIRE].

[78] C. Englert, Y. Soreq and M. Spannowsky, Off-Shell Higgs Coupling Measurements in BSM scenarios, arXiv:1410.5440 [INSPIRE].

[79] A. Devastato, F. Lizzi, C.V. Flores and D. Vassilevich, Unification of Coupling Constants, Dimension six Operators and the Spectral Action, Int. J. Mod. Phys. A 30 (2015) 1550033 [arXiv: 1410.6624] [INSPIRE].

[80] S. Willenbrock and C. Zhang, Effective Field Theory Beyond the Standard Model, Ann. Rev. Nucl. Part. Sci. 64 (2014) 83 [arXiv:1401.0470] [INSPIRE].

[81] F. del Aguila and J. de Blas, Electroweak constraints on new physics, Fortsch. Phys. 59 (2011) 1036 [arXiv:1105.6103] [INSPIRE].

[82] J. de Blas, Electroweak limits on physics beyond the Standard Model, EPJ Web Conf. 60 (2013) 19008 [arXiv:1307.6173] [INSPIRE].

[83] J. de Blas et al., Global Bayesian Analysis of the Higgs-boson Couplings, arXiv:1410.4204 [INSPIRE].

[84] T. Corbett, O.J.P. Eboli, J. Gonzalez-Fraile and M.C. Gonzalez-Garcia, Robust Determination of the Higgs Couplings: Power to the Data, Phys. Rev. D 87 (2013) 015022 [arXiv: 1211.4580] [INSPIRE].

[85] B. Dumont, S. Fichet and G. von Gersdorff, A Bayesian view of the Higgs sector with higher dimensional operators, JHEP 07 (2013) 065 [arXiv: 1304.3369] [INSPIRE].

[86] Z. Han and W. Skiba, Effective theory analysis of precision electroweak data, Phys. Rev. D 71 (2005) 075009 [hep-ph/0412166] [INSPIRE]. 
[87] M. Ciuchini, E. Franco, S. Mishima and L. Silvestrini, Electroweak Precision Observables, New Physics and the Nature of a 126 GeV Higgs Boson, JHEP 08 (2013) 106 [arXiv: 1306.4644] [INSPIRE].

[88] A. Pomarol and F. Riva, Towards the Ultimate SM Fit to Close in on Higgs Physics, JHEP 01 (2014) 151 [arXiv: 1308.2803] [INSPIRE].

[89] J. Ellis, V. Sanz and T. You, Complete Higgs Sector Constraints on Dimension-6 Operators, JHEP 07 (2014) 036 [arXiv: 1404.3667] [INSPIRE].

[90] ALEPH, DELPHI, L3, OPAL, SLD collaborations, the LEP Electroweak Working Group, the SLD Electroweak Group, the SLD Heavy Flavour Group, S. Schael et al., Precision electroweak measurements on the $Z$ resonance, Phys. Rept. 427 (2006) 257 [hep-ex/0509008] [INSPIRE].

[91] D0 collaboration, V.M. Abazov et al., Combined search for the standard model Higgs boson

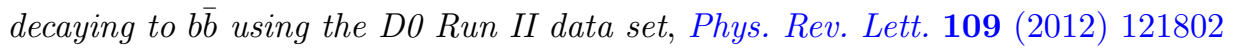
[arXiv: 1207.6631] [INSPIRE].

[92] CMS collaboration, Observation of a new boson with mass near $125 \mathrm{GeV}$ in pp collisions at $\sqrt{s}=7$ and $8 \mathrm{TeV}$, JHEP 06 (2013) 081 [arXiv: 1303.4571] [INSPIRE].

[93] ATLAS collaboration, Constraints on New Phenomena via Higgs Coupling Measurements with the ATLAS Detector, ATLAS-CONF-2014-010 (2014).

[94] ATLAS collaboration, Search for the bb decay of the Standard Model Higgs boson in associated W/ZH production with the ATLAS detector, ATLAS-CONF-2013-079 (2013).

[95] CMS collaboration, Measurement of the $W^{+} W^{-}$Cross section in pp Collisions at $\sqrt{s}=7 \mathrm{TeV}$ and Limits on Anomalous $W W \gamma$ and $W W Z$ couplings, Eur. Phys. J. C 73 (2013) 2610 [arXiv: 1306.1126] [inSPIRE].

[96] CMS collaboration, Measurement of $W^{+} W^{-}$and $Z Z$ production cross sections in $p p$ collisions at $\sqrt{s}=8 \mathrm{TeV}$, Phys. Lett. B 721 (2013) 190 [arXiv:1301.4698] [inSPIRE].

[97] ATLAS collaboration, Measurement of the $W^{+} W^{-}$production cross section in proton-proton collisions at $\sqrt{s}=8 \mathrm{TeV}$ with the ATLAS detector, ATLAS-CONF-2014-033 (2014).

[98] A. Falkowski, S. Fichet, K. Mohan, F. Riva and V. Sanz, Triple gauge couplings revisited, to appear.

[99] M.E. Peskin and T. Takeuchi, A new constraint on a strongly interacting Higgs sector, Phys. Rev. Lett. 65 (1990) 964 [INSPIRE].

[100] M.E. Peskin and T. Takeuchi, Estimation of oblique electroweak corrections, Phys. Rev. D 46 (1992) 381 [INSPIRE].

[101] I. Maksymyk, C.P. Burgess and D. London, Beyond S, T and U, Phys. Rev. D 50 (1994) 529 [hep-ph/9306267] [INSPIRE].

[102] R. Barbieri, A. Pomarol, R. Rattazzi and A. Strumia, Electroweak symmetry breaking after LEP-1 and LEP-2, Nucl. Phys. B 703 (2004) 127 [hep-ph/0405040] [INSPIRE].

[103] G. Altarelli and R. Barbieri, Vacuum polarization effects of new physics on electroweak processes, Phys. Lett. B 253 (1991) 161 [INSPIRE].

[104] R. Barbieri and A. Strumia, What is the limit on the Higgs mass?, Phys. Lett. B 462 (1999) 144 [hep-ph/9905281] [INSPIRE]. 
[105] R. Contino, M. Ghezzi, C. Grojean, M. Muhlleitner and M. Spira, Effective Lagrangian for a light Higgs-like scalar, JHEP 07 (2013) 035 [arXiv:1303.3876] [INSPIRE].

[106] J.D. Wells and Z. Zhang, Precision Electroweak Analysis after the Higgs Boson Discovery, Phys. Rev. D 90 (2014) 033006 [arXiv:1406.6070] [InSPIRE].

[107] R.S. Gupta, A. Pomarol and F. Riva, BSM Primary Effects, Phys. Rev. D 91 (2015) 035001 [arXiv: 1405.0181] [INSPIRE].

[108] D.Y. Bardin et al., ZFITTER v.6.21: A semianalytical program for fermion pair production in $e^{+} e^{-}$annihilation, Comput. Phys. Commun. 133 (2001) 229 [hep-ph/9908433] [INSPIRE].

[109] M. Baak et al., The Electroweak Fit of the Standard Model after the Discovery of a New Boson at the LHC, Eur. Phys. J. C 72 (2012) 2205 [arXiv:1209.2716] [INSPIRE].

[110] C. Grojean, E.E. Jenkins, A.V. Manohar and M. Trott, Renormalization Group Scaling of Higgs Operators and $\Gamma(h \rightarrow \gamma \gamma)$, JHEP 04 (2013) 016 [arXiv:1301.2588] [INSPIRE].

[111] J. Elias-Miró, J.R. Espinosa, E. Masso and A. Pomarol, Renormalization of dimension-six operators relevant for the Higgs decays $h \rightarrow \gamma \gamma, \gamma Z$, JHEP 08 (2013) 033 [arXiv:1302.5661] [INSPIRE].

[112] J. Elias-Miro, J.R. Espinosa, E. Masso and A. Pomarol, Higgs windows to new physics through $D=6$ operators: constraints and one-loop anomalous dimensions, JHEP 11 (2013) 066 [arXiv: 1308.1879] [INSPIRE].

[113] E.E. Jenkins, A.V. Manohar and M. Trott, Renormalization Group Evolution of the Standard Model Dimension Six Operators I: Formalism and lambda Dependence, JHEP 10 (2013) 087 [arXiv: 1308.2627] [INSPIRE].

[114] E.E. Jenkins, A.V. Manohar and M. Trott, Renormalization Group Evolution of the Standard Model Dimension Six Operators II: Yukawa Dependence, JHEP 01 (2014) 035 [arXiv: 1310.4838] [INSPIRE].

[115] R. Alonso, E.E. Jenkins, A.V. Manohar and M. Trott, Renormalization Group Evolution of the Standard Model Dimension Six Operators III: Gauge Coupling Dependence and Phenomenology, JHEP 04 (2014) 159 [arXiv: 1312.2014] [INSPIRE].

[116] J. Elias-Miró, C. Grojean, R.S. Gupta and D. Marzocca, Scaling and tuning of EW and Higgs observables, JHEP 05 (2014) 019 [arXiv: 1312.2928] [INSPIRE].

[117] R. Alonso, H.-M. Chang, E.E. Jenkins, A.V. Manohar and B. Shotwell, Renormalization group evolution of dimension-six baryon number violating operators, Phys. Lett. B 734 (2014) 302 [arXiv: 1405. 0486] [INSPIRE].

[118] A. Alloul, B. Fuks and V. Sanz, Phenomenology of the Higgs Effective Lagrangian via FeynRules, JHEP 04 (2014) 110 [arXiv:1310.5150] [INSPIRE].

[119] M. Trott, On the consistent use of Constructed Observables, JHEP 02 (2015) 046 [arXiv: 1409.7605] [INSPIRE].

[120] P. Artoisenet et al., A framework for Higgs characterisation, JHEP 11 (2013) 043 [arXiv: 1306.6464] [INSPIRE].

[121] F. Demartin, F. Maltoni, K. Mawatari, B. Page and M. Zaro, Higgs characterisation at NLO in QCD: CP properties of the top-quark Yukawa interaction, Eur. Phys. J. C 74 (2014) 3065 [arXiv: 1407.5089] [INSPIRE]. 
[122] F. Maltoni, K. Mawatari and M. Zaro, Higgs characterisation via vector-boson fusion and associated production: NLO and parton-shower effects, Eur. Phys. J. C 74 (2014) 2710 [arXiv: 1311.1829] [INSPIRE].

[123] LHC Higgs Cross section Working Group collaboration, A. David et al., LHC HXSWG interim recommendations to explore the coupling structure of a Higgs-like particle, arXiv: 1209.0040 [INSPIRE].

[124] J. Alwall, M. Herquet, F. Maltoni, O. Mattelaer and T. Stelzer, MadGraph 5: Going Beyond, JHEP 06 (2011) 128 [arXiv:1106.0522] [INSPIRE].

[125] T. Sjöstrand, S. Mrenna and P.Z. Skands, PYTHIA 6.4 Physics and Manual, JHEP 05 (2006) 026 [hep-ph/0603175] [INSPIRE].

[126] DELPHES 3 collaboration, J. de Favereau et al., DELPHES 3, A modular framework for fast simulation of a generic collider experiment, JHEP 02 (2014) 057 [arXiv:1307.6346] [INSPIRE].

[127] A. Biekoetter, A. Knochel, M. Kraemer, D. Liu and F. Riva, Vices and Virtues of Higgs EFTs at Large Energy, arXiv:1406.7320 [INSPIRE].

[128] M. Beneke, D. Boito and Y.-M. Wang, Anomalous Higgs couplings in angular asymmetries of $H \rightarrow Z \ell^{+} \ell^{-}$and $e^{+} e^{-} \rightarrow H Z$, JHEP 11 (2014) 028 [arXiv:1406.1361] [InSPIRE].

[129] B. Henning, X. Lu and H. Murayama, What do precision Higgs measurements buy us?, arXiv: 1404.1058 [INSPIRE].

[130] M. Gorbahn, J.M. No and V. Sanz, Benchmarks for Higgs Effective Theory: Extended Higgs Sectors, arXiv:1502.07352 [INSPIRE].

[131] J.F. Gunion and H.E. Haber, The CP conserving two Higgs doublet model: The approach to the decoupling limit, Phys. Rev. D 67 (2003) 075019 [hep-ph/0207010] [INSPIRE].

[132] M. Carena, I. Low, N.R. Shah and C.E.M. Wagner, Impersonating the Standard Model Higgs Boson: Alignment without Decoupling, JHEP 04 (2014) 015 [arXiv:1310.2248] [INSPIRE].

[133] E. Massó and V. Sanz, Limits on anomalous couplings of the Higgs boson to electroweak gauge bosons from LEP and the LHC, Phys. Rev. D 87 (2013) 033001 [arXiv:1211.1320] [INSPIRE].

[134] A. Djouadi, V. Driesen, W. Hollik and A. Kraft, The Higgs photon-Z boson coupling revisited, Eur. Phys. J. C 1 (1998) 163 [hep-ph/9701342] [INSPIRE]. 